\title{
INFLUENCIA DE LA CIRCULACIÓN EÓLICA Y MARÍTIMA EN LA FORMACIÓN DE LAS ISLAS DE BASURA EN EL MUNDO
}

Influence of wind and ocean circulation in the formation of the islands of garbage in the world

\section{Martín A. Meléndez Valencia Patricia Isabel Meléndez Torres}

Resumen: Las circulaciones marinas son una consecuencia directa de la convección que se produce entre la tierra, la capa límite de la atmósfera y los océanos responde al patrón general de la circulación atmosférica. El viento sobre la superficie del océano es el motor de las grandes corrientes oceánicas superficiales que conocemos. Estas corrientes van recogiendo todo tipo de basura en su trayectoria y al final forman lo que se conoce como "Islas de Basura".

La mayor de las islas, la del pacífico, está situada a unos 1.000 kilómetros de Hawái y tiene 3.4 millones de $\mathrm{km}^{2}$. La isla de basura del Caribe está ubicada aproximadamente a los 21 grados de latitud norte, 65 longitud oeste.

Estos residuos afectan por lo menos 267 especies alrededor del mundo. La basura ha destrozado un tercio de la superficie de hábitats marinos y se estima que el 35\% de los ecosistemas marinos críticos han sido destruidos.

A diferencia de los desechos biodegradables, los plásticos fotodegradables se desintegran en pedazos más pequeños, aunque permanecen siendo polímeros. Este proceso continúa hasta llegar 
a nivel molecular. Las concentraciones de plástico se acercaban a una parte de millón por millas cuadradas. El estudio encontró concentraciones de plástico de 3,3 partes con una masa de 5,1 miligramos por metro cuadrado.

Palabras claves: Islas de basuras, efecto de Coriolis, Espiral de Ekman, circulación eólica.

Abstract: The marine circulations are a direct consequence of convection that occurs between the earth, the boundary layer of the atmosphere and oceans respond to the general pattern of atmospheric circulation. The wind on the surface of the ocean is the engine of the great ocean currents surface we know. These currents are collecting all kinds of junk in its path and eventually form what is known as "Garbage Islands".

The largest of the islands, the Pacific is located about 1,000 miles from Hawaii, 3.4 million $\mathrm{km}^{2}$. The Caribbean island of trash is located approximately 21 degrees north latitude, 65 west longitude.

These residues affect at least 267 species worldwide. The garbage has destroyed a third of the surface of marine habitats and it is estimated that $35 \%$ of critical marine ecosystems have been destroyed. Unlike biodegradable waste, photodegradable plastics disintegrate into smaller pieces, but remain still polymers. This process continues down to the molecular level. The Plastic concentrations approaching one part per million per square mile. The study found plastic concentrations of 3.3 parts with a mass of 5.1 milligrams per square meter.

Keywords: Islands of garbage, Coriolis Effect, Ekman spiral, wind circulation. 


\section{Introducción}

Desde que Jean Baptista Joseph Fourier, matemático francés, observó que ciertos gases, en particular el dióxido de carbono, retienen el "calor atmosférico $\mathrm{CO}_{2}$ " dentro de los invernaderos; y posteriormente, desde que el premio Nobel sueco (1903) Svante August Arrhenius construyó la teoría general de efecto invernadero que causaba el calentamiento del planeta; la Mecánica de Fluidos y el medio ambiente iniciaron -como parece de hechouna fructífera colaboración que dura hasta nuestros días.

La Mecánica de Fluidos influye en el medio ambiente y en las técnicas de control y medición de contaminantes que se centran en el área de la atmósfera, alta y baja (estratósfera y tropósfera) de la Tierra.

Si describimos la atmósfera, nos adentramos en la Mecánica de Fluidos. ¿Por qué decimos que la atmósfera tiene que ver con la mecánica de fluidos? -Porque si hablamos de la contaminación atmosférica, esta se desplaza según la dinámica de la propia atmósfera y según las leyes de traslación de la dinámica de fluidos.

Un ejemplo lo tenemos en la catástrofe de Chernóbil, sucedida en Rusia, pero que a los pocos días se detecta en Escocia, como consecuencia de un movimiento de masas de aire. De modo que conocer la dinámica de la baja atmósfera -aquella que va desde el suelo hasta los 10,000 m. de altura- es fundamental para entender y predecir accidentes en nuestro medioambiente.

En la actualidad, la contaminación atmosférica está "globalizada": puede existir un crecimiento del agujero de Ozono localizado en el centro de Europa ${ }^{1}$ que, en pocas semanas, se desplace hasta el hemisferio Sur.

1 En el Hemisferio Sur es donde este fenómeno se manifiesta de manera más contundente. 


\section{Flujos atmosféricos}

El entendimiento de los flujos del viento y los procesos de transporte atmosféricos es crucial, por ejemplo, para predecir y cuantificar la dispersión de los contaminantes en la atmósfera; también permiten calcular el efecto invernadero y predecir el clima.

La atmósfera se conforma de dos capas básicas: la tropósfera y la estratósfera. La diferencia entre ambas es que en la tropósfera, la temperatura disminuye en forma lineal con la altura (está pendiente de la curva se llama "rapidez de descenso"), mientras que en la estratósfera, la temperatura permanece más o menos constante con la altura.

Figura N. ${ }^{\circ} 1$

Variación de la temperatura con la altura en la atmósfera estándar de Estados Unidos

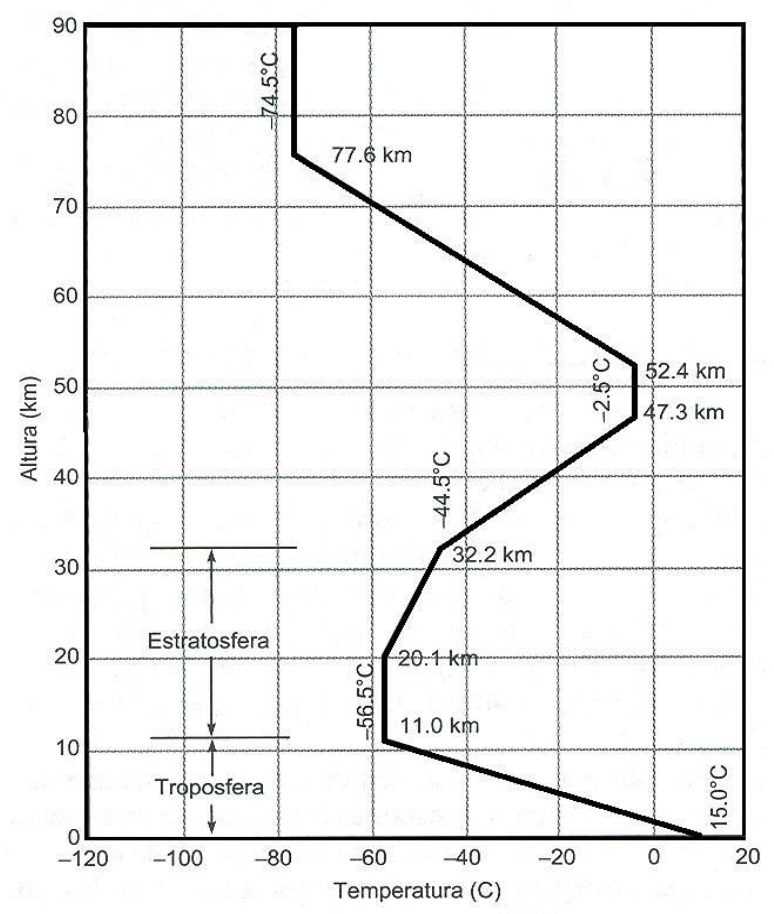

Fuente: The U.S. Standard Atmosphere, U.S. Government Printing Office (1976) 
La rapidez de descenso y la altura de la tropósfera varían con el tiempo y la posición, pero de acuerdo al Servicio del Clima de Estados Unidos, que ha reunido una serie de datos promedios durante los últimos 200 años, ellos identifican el punto ubicado a los $40^{\circ}$ de latitud norte como el punto donde está ubicada la "Atmósfera Estándar de Estados Unidos".

En consonancia con esta atmósfera estándar -que no necesariamente proporciona una descripción exacta de la atmósfera en cualquier posición o instante- la tropósfera se extiende desde el nivel del mar hasta una altura de $11,000 \mathrm{~m}$, con una rapidez de descenso en su temperatura de $6.5^{\circ} \mathrm{K}$ por cada $1,000 \mathrm{~m}$ de descenso. Esto también se conoce como "Rapidez de Descenso Estándar”.

La estratósfera está por encima de la tropósfera y se extiende hasta una altura de $32.2 \mathrm{~km}$ y su temperatura es constante a $216.7 \mathrm{~K}\left(-56.5^{\circ} \mathrm{C}\right)$ a $20.1 \mathrm{~km}$ de altura. ${ }^{2}$

La tropósfera contiene entre un 80 y un $85 \%$ de la masa total de la atmósfera y, de hecho, toda el agua de la atmósfera, por lo que tiene la función más importante en la determinación del clima y el estado del tiempo.

Por encima de los $20.1 \mathrm{~km}$, la temperatura aumenta de forma gradual con la altura, debido a que el ozono absorbe la radiación infrarroja solar, el cual se forma con la intensa radiación ultravioleta del Sol. Esta absorción del ozono también protege la vida sobre la tierra de los efectos destructivos de los rayos ultravioletas.

2 Algunos autores consideran que la estratósfera se extiende solo arriba de los 20.1 $\mathrm{km}$, de manera que se confina a la región de la temperatura constante. 


\section{Equilibrio de la atmósfera ${ }^{3}$}

La tropósfera (la parte baja de la atmósfera), se mezcla de manera continua por la convección. ${ }^{4} \mathrm{El}$ vapor de agua se eleva y luego se precipita y existen grandes movimientos de viento polar y tropical hacia las regiones templadas.

En los trópicos, debido al intenso calentamiento del suelo, el aire se eleva y esto produce una circulación a gran escala desde las regiones templadas hacia los trópicos y una tendencia cerca de la cima de la tropósfera desde los trópicos hacia los polos.

Como resultado de todo lo anterior, la tropósfera mantiene un equilibrio térmico y mecánico constante más o menos estable, que es lo que determina el clima.

Las desviaciones de este equilibrio es lo que se conoce como clima, y este varía de un día a otro y hasta de una hora a otra, de manera irregular.

\subsection{LA CONVECCIÓN EN LA ATMÓSFERA}

La convección es una de las tres formas de transferencia de calor y se caracteriza porque se produce por intermedio de un fluido (aire y/o agua) que transporta el calor entre zonas con diferentes temperaturas.

La convección se produce únicamente por medio de fluidos. Al calentarse estos, aumenta de volumen y, por tanto, su densidad

3 El material de esta sección es una adaptación-interpretación del libro Engineering Applications of Fluids Mechanics, de Hunsaker y Rightmire (1947).

4 La convección tiene lugar cuando áreas de fluido caliente (de menor densidad) ascienden hacia las regiones de fluido frío. Cuando ocurre esto, el fluido frío (de mayor densidad) desciende y ocupa el lugar del fluido caliente que ascendió. Este ciclo da lugar a una continua circulación (corrientes convectivas) del calor hacia las regiones frías. 
disminuye y ascienden desplazando el fluido que se encuentra en la parte superior y que está a menor temperatura. Lo que se llama convección en sí es el transporte de calor por medio de las corrientes ascendentes y descendentes del fluido.

La transferencia de calor por convección se expresa con la Ley del enfriamiento de Newton:

$$
\frac{d Q}{d t}=h A_{s}\left(T_{s}-T_{i n f}\right)
$$

Donde $\mathrm{h}$ es el coeficiente de convección (o coeficiente de película); $A_{s}$ es el área del cuerpo en contacto con el fluido, $T_{S}$ es la temperatura en la superficie del cuerpo y $T_{\text {inf }}$ es la temperatura del fluido lejos del cuerpo.

La convección en la atmósfera terrestre involucra la transferencia de enormes cantidades del calor absorbido por el agua. Forma nubes de gran desarrollo vertical (por ejemplo, cúmulos congestus y, sobre todo, cumulonimbos, que son los tipos de nubes que alcanzan mayor desarrollo vertical).

Estas nubes son las típicas portadoras de tormentas eléctricas y de grandes chaparrones. $\mathrm{Al}$ alcanzar una altura muy grande (por ejemplo, unos 12 o $14 \mathrm{~km}$ ) y enfriarse violentamente, pueden producir tormentas de granizo, ya que las gotas de lluvia se van congelando al ascender violentamente y luego se precipitan al suelo ya en estado sólido. Pueden tener forma de un hongo asimétrico de gran tamaño; y a veces se forma en este tipo de nubes una estela que semeja una especie de yunque (anvil's head, como se conoce en inglés).

El proceso que origina la convección en el seno de la atmósfera es sumamente importante y genera una serie de fenómenos fundamentales en la explicación de los vientos y en la formación de nubes, vaguadas, ciclones, anticiclones, precipitaciones, etc. 
Todos los procesos y mecanismos de convección del calor atmosférico obedecen a las leyes físicas de la termodinámica. De estos procesos es fundamental el que explica el ciclo del agua en la naturaleza o ciclo hidrológico. Casi todos los fenómenos antes nombrados tienen que ver con este último mecanismo.

El clima está determinado por el estado del tiempo promedio en un lugar dado en cierta época del año, sobre muchas estaciones $y$, por ejemplo, la climatología con base en registros anteriores predice la fecha segura para plantar cultivos.

La naturaleza fundamental de la convección sugiere que la atmósfera más baja debería tener un gradiente de temperatura más o menos adiabático. El aire cercano al suelo que se calienta más en un lado que en otro subirá como el aire tibio en una chimenea. El aire más frío y más denso fluirá para tomar su lugar.

El aire ascendente se enfriará casi en forma adiabática, pues es mal conductor. Para encontrar la rapidez de enfriamiento se inicia con la ecuación de la hidrostática $(d p / d z=-\rho g)$ y se usan las relaciones termodinámicas para el flujo adiabático no viscoso (o flujo isentrópico5).

Para poder obtener la rapidez de descenso adiabático:

$$
\text { Rapidez de descenso adiabático }=\left(\frac{d T}{d z}\right)_{a d}=\frac{\gamma-1}{\gamma R} g^{6}
$$

Si observamos la rapidez del descenso natural de la temperatura, observaremos que esta contribuye a estabilizar la atmósfera más baja; es decir, una masa de aire que se desplaza en forma vertical, por alguna razón tiende a volver a su nivel original.

\footnotetext{
5 Un proceso isentrópico es aquel en el que la entropía del fluido, que forma el sistema, permanece constante.

6 Que para el aire seco es igual a $0.0098^{\circ} \mathrm{C} / \mathrm{m}$.
}

750 Ciencia y Sociedad 2013; 38(4): 743-792 


\subsection{COMPORTAMIENTO DE UN FLUIDO CUALQUIERA EN LA TRANSFERENCIA DE CALOR}

Cuando un fluido cede calor, sus moléculas se desaceleran por lo cual su temperatura disminuye y su densidad aumenta siendo atraída sus moléculas por la gravedad de la tierra.

Cuando el fluido absorbe calor sus moléculas se aceleran por lo cual su temperatura aumenta y su densidad disminuye haciéndolo más liviano. El fluido más frío tiende a bajar y ocupa el nivel más bajo de la vertical y los fluidos más calientes son desplazados al nivel más alto, creándose así los vientos de la tierra.

La transferencia térmica convectiva consiste en el contacto del fluido con una temperatura inicial con otro elemento o material con una temperatura diferente, en función de la variación de las temperaturas van a variar las cargas energéticas moleculares del fluido y los elementos inter-actuantes del sistema realizarán un trabajo, donde el que tiene mayor energía o temperatura se la cederá al que tiene menos temperatura. Esta transferencia térmica se realizará hasta que los dos tengan igual temperatura, mientras se realiza el proceso las moléculas con menor densidad tienden a subir y las de mayor densidad a bajar de nivel.

En general, el aire es estable y la convección no sucede a gran escala. Sin embargo, cuando la rapidez de descenso atmosférico excede la rapidez adiabática, el aire es inestable. Los truenos de las tormentas se dan en atmósferas inestables, donde la rapidez de descenso es excesiva temporalmente por los calentamientos anormales del suelo, y de los niveles más bajos del aire. Esta condición suele suceder en verano. En invierno, cuando el suelo puede cubrirse de nieve, los niveles más bajos están fríos, la rapidez de descenso es pequeña y el aire tiende a ser estable.

En una noche de verano, el suelo se enfría rápido por radiación y el nivel más bajo del aire puede hacerse más frío que el de encima. 
Aquí la rapidez de descenso puede ser cero o aún revertirse. A esto se llama inversión térmica y aporta una gran estabilidad.

La formación de cúmulos de nubes y por lo tanto de tormentas eléctricas es el resultado de la inestabilidad del aire.

Este aire tibio, húmedo cerca del suelo se vuelve inestable y se eleva, enfriándose de forma adiabática hasta alcanzar el punto de rocío, cuando la condensación del vapor de agua forma una nube (Véase la figura N. ${ }^{\circ}$ ). ${ }^{7}$

\section{Figura N. ${ }^{\circ} 2$}

Formación de una nube por condensación del vapor de agua arrastrado hacia arriba por una corriente de aire

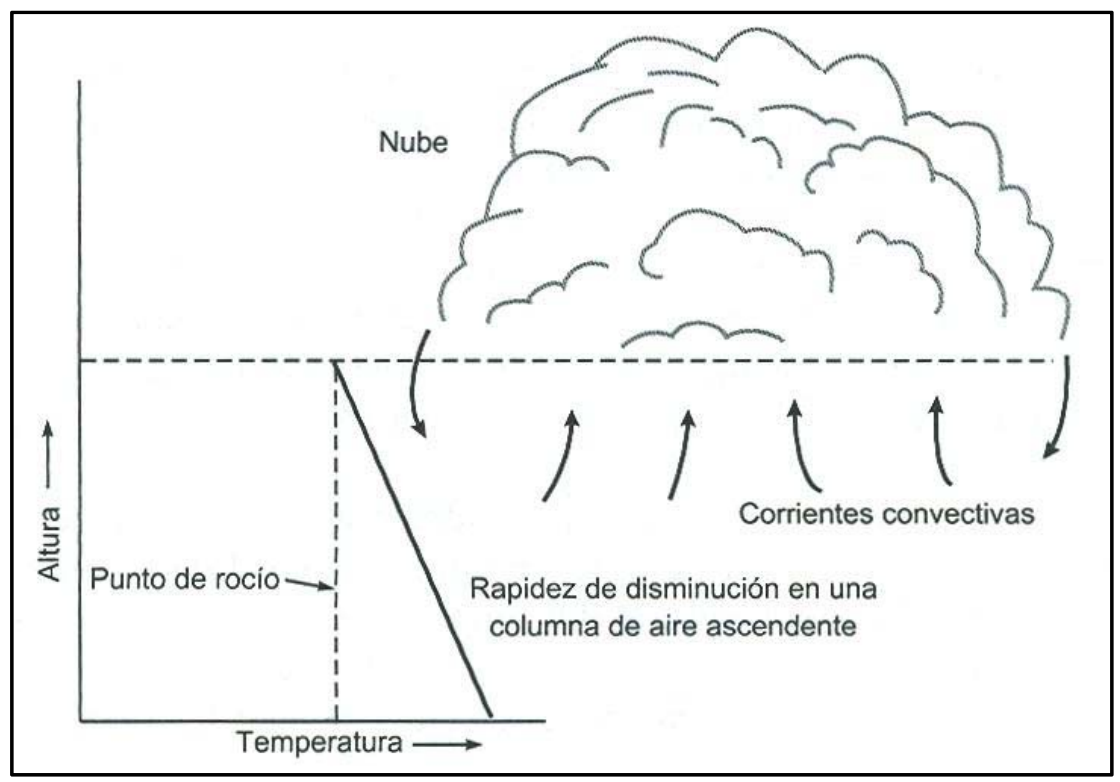

Formación de una nube por condensación del vapor de agua arrastrado hacia arriba por una corriente de aire. (Véase Hunsaker y Rightmire, 1986). 
Si el despegue inicial es violento, el aire ascendente puede sobrepasar su altura de equilibrio. Si a esta altura hay una inversión o una capa de aire estable con ella se detiene las subsecuentes corrientes ascendentes.

Sin embargo, si el aire superior es neutro o ligeramente inestable, el calor latente que se libera por la condensación puede ser suficiente para arrastrar la corriente convectiva hasta alturas mayores. Como ya explicamos, las nubes de las tormentas se extienden hasta los 50,000 pies, aunque el aire es limpio por encima de los 20,000 pies.

El movimiento del aire es siempre turbulento al igual que el de las nubes. El número de Reynolds se basa en una dimensión característica aproximada de la nube, como la altura o la anchura (en general ambas son del mismo orden). Con una nube de alrededor de $500 \mathrm{~m}$ y un movimiento interno característico de $5 \mathrm{~m} / \mathrm{s}$ y con una $v=10^{-5} \mathrm{~m}^{2} / \mathrm{s}$ (es la misma viscosidad cinemática que para el vapor de agua que para el aire) el número de Reynolds sea de $2.5 \times 10^{8}$, lo que nos indica que es turbulencia completa.

No es sorpresa que las nubes siempre tengan una apariencia turbulenta.

\section{Patrones circulatorios y efectos de Coriolis}

El sol, la tierra y la atmósfera terrestre forman un sistema dinámico muy grande. El calentamiento diferencial del aire da lugar a gradientes de presión horizontales que, a su vez, producen movimientos horizontales en la atmósfera. ${ }^{8}$

La diferencia de temperatura entre la atmósfera de los polos y del ecuador y entre la atmósfera de los continentes y los océanos

${ }^{8}$ Air pollution: Its origin and control. Fuente: Wark, Warner y Davis (1998). 
causan movimientos de gran escala de la atmósfera. Los vientos locales, así como las brisas de los lagos, también se originan por las diferencias de temperaturas.

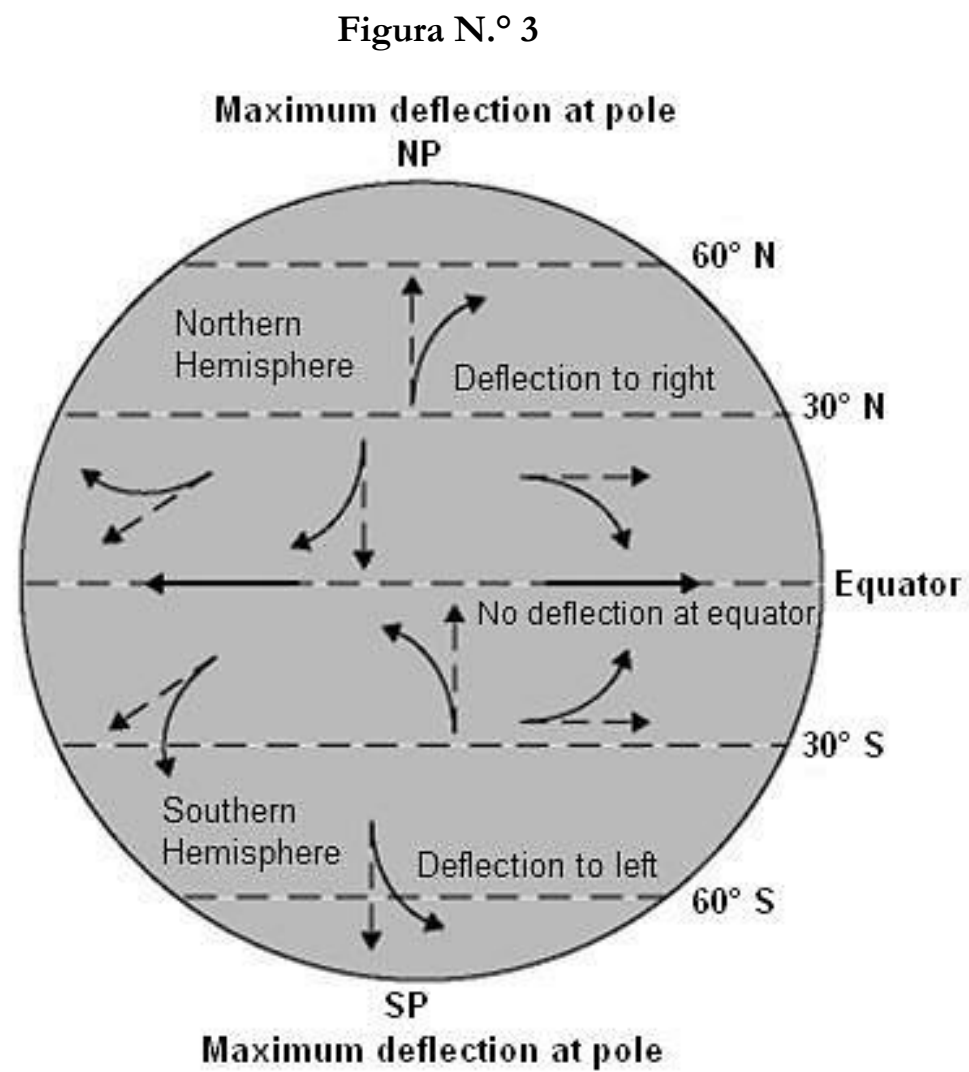

Fuente: Extraído de Smits (2005).

Una superficie de tierra se calienta y enfría más rápido por radiación que un cuerpo grande de agua, por lo que durante el día el aire tiende a subir desde la tierra, causando que el aire se mueva desde el agua hacia la tierra. Durante la noche, la tierra se enfría más rápidamente, de modo que el aire sobre ella poco a poco se hace mas frío y denso que en el agua y el movimiento del aire es de la tierra al agua. 
Si la tierra no rotara, el aire tendería a fluir de manera natural en dirección del gradiente de presión, o sea, desde las regiones de presión mayor hacia las de presión más baja. El flujo sería perpendicular a las isóbaras (contornos de presión constante).

Sin embargo, la rotación de la tierra impide que esto suceda, al menos en la escala de los grandes patrones del clima.

Para un observador colocado en un punto de referencia rotatorio, como la tierra, existen fuerzas aparentes que pueden actuar, además de los efectos de los gradientes de presión, la gravedad, las fuerzas viscosas; la más importante de todas es la fuerza de Coriolis.

El sistema de referencia "más apropiado" es el de las estrellas distantes. En la superficie terrestre, en realidad, se acelera por el movimiento alrededor del sol, el giro de la tierra alrededor de su eje de rotación y por otros movimientos que no se logran detectar. ${ }^{9}$

El más importante de estos movimientos es el giro alrededor de su eje, que es 365 veces mayor que el de la velocidad angular alrededor del sol. De esta manera, el centro de la tierra, nos sirve de un excelente marco de referencia inercial. ([X,Y,Z]).

Las posiciones, velocidades y aceleraciones que se observan (fijas sobre la superficie terrestre) no son las que ve un observador fijo respecto al centro de la tierra. Para muchas aplicaciones, la diferencia entre los dos sistemas de referencia es despreciable.

Estas diferencias se hacen nobles solo en escala grande como son los movimientos atmosféricos y los oceánicos.

${ }^{9}$ Véase a Smits (2005). Una introducción física a la mecánica de fluidos. 


\section{Figura N. ${ }^{\circ} 4$}

Sistema de coordenadas para la rotación de la Tierra alrededor de su eje XYZ es el sistema de coordenado inercial en el centro terrestre; $x y z$ es el sistema de coordenadas local en la latitud $\beta$ (en el ecuador, la latitud es cero), $R$ es el radio de la Tierra

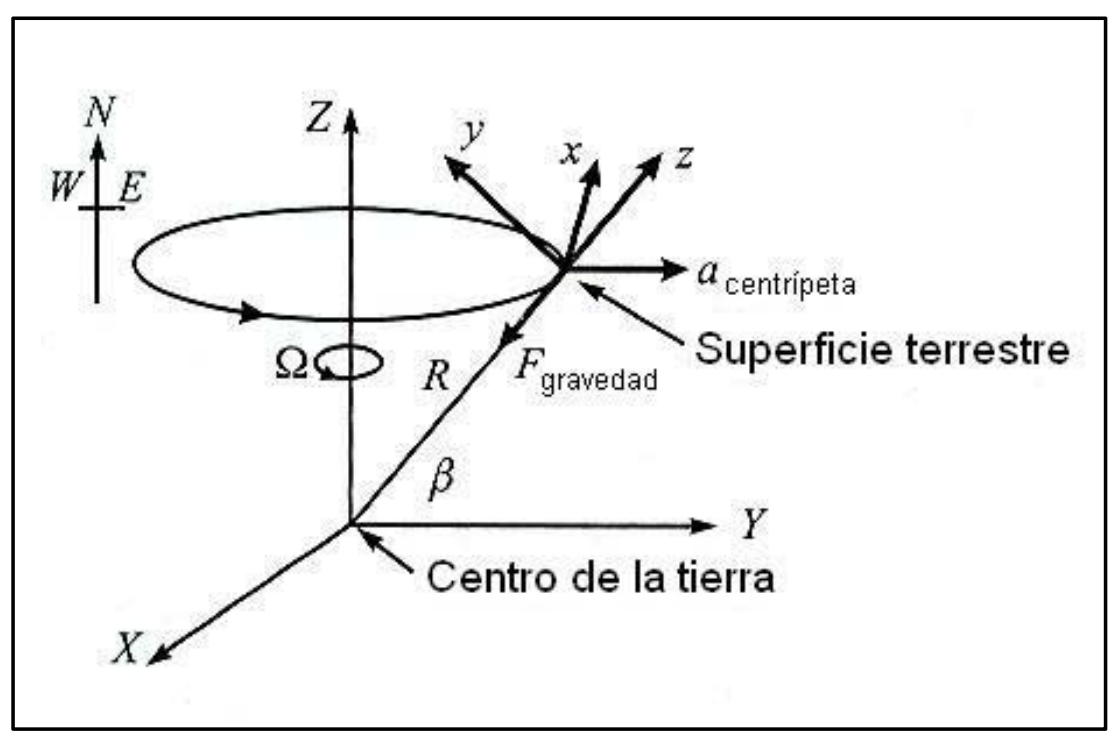

Si se utilizan las ecuaciones de Euler es posible desarrollar expresiones para las ecuaciones de movimiento con respecto a la superficie terrestre. Es de sumar un término a la ecuación de Euler (que representa una aceleración "aparente"): hay que tratarla tanto matemática y conceptualmente como si fuera una fuerza.

La tierra gira sobre su eje de oeste a este, de manera que si se observa sobre el polo norte habrá una dirección en el sentido contrario de las manecillas del reloj.

Si el vector de velocidad angular de la rotación de la tierra es $\Omega \rightarrow$, que es igual a la rotación angular de una partícula de fluido en cualquier latitud, $\beta$, que se sabe que

$$
\Omega=2 \pi \mathrm{rad} / \text { día }
$$


La cantidad de movimiento angular de la partícula por unidad de masa relativa al sistema de referencia inercial (el centro de la tierra) será

$$
r^{*} V
$$

Donde $r=$ radio de rotación

$$
V=\text { Velocidad }
$$

$$
\text { La velocidad } \quad V=\Omega \rightarrow * r
$$

Como $r$ se mide desde el eje de rotación,

$$
r=R \cos \beta
$$

donde $\mathrm{R}$ es el radio de la Tierra (= $6380 \mathrm{~km})$.

Una partícula en el hemisferio norte, que se mueve en dirección al polo norte, lo hará hacia el eje de rotación. En ausencia de fricción, la cantidad de movimiento angular se conserva y el fluido adquiere una mayor velocidad angular, ya que el radio disminuye $\mathrm{y}$ aumenta $\beta$. (Este $\beta$ es el efecto que se observa cuando al girar, un patinador levanta los brazos para incrementar su rapidez de rotación).

Así una partícula en el hemisferio norte se desviará hacia la derecha (el este) y una partícula en el sur requiere de una velocidad angular menor para conservar la cantidad de movimiento angular y se desvía hacia el oeste.

Consideremos, por otra parte, movimientos en dirección esteoeste a una latitud constante. Una partícula en el hemisferio norte que se mueve hacia el este aumentará su velocidad angular y entonces para conservar su cantidad de movimiento angular, experimentará una tendencia a moverse hacia un radio mayor de rotación y se desviará hacia el sur. 
De manera semejante, una partícula del oeste tendrá una velocidad angular menor y experimentará una tendencia a desviarse al norte para conservar la cantidad de movimiento angular.

Los resultados globales de estos efectos de Coriolis son crear patrones de movimiento circulatorio en el sentido de las manecillas del reloj en el hemisferio norte y patrones de movimiento circulatorio en dirección contraria de las manecillas del reloj en el hemisferio sur. Esto es irrefutable en la atmósfera y para los océanos.

Los efectos de Coriolis son los mecanismos dominantes que controlan estos patrones circulatorios y, por tanto, efectivamente controlan los patrones del clima.

La fuerza de Coriolis, una fuerza aparente, se presenta en las ecuaciones de movimiento debido a la rotación de la Tierra. Dado que las velocidades son, en general, mucho más pequeñas que las velocidades en el plano horizontal, se tiene:

$$
2 \vec{\Omega} \times \mathrm{V}=-\mathrm{fvi}+\mathrm{fuj}
$$

Donde $f$ es el parámetro de Coriolis, el cual podemos definir como:

$$
f=2 \Omega \operatorname{sen} \beta \quad 0 \leq f \leq 1.44 \times 10^{-4} s^{-1}
$$

Sobre la tierra el valor de $f$ variará entre los siguientes valores.

Si adimensionalizamos la ecuación

$$
\frac{D V}{D t}+2 \vec{\Omega} \times V=-\frac{1}{\rho} \nabla_{p-g}
$$

Veremos que el término de Coriolis se convierte:

$$
\left(\frac{2 \operatorname{sen} \beta}{R o}\right) V^{\prime}
$$


Donde $V^{\prime}$ es la velocidad adimensional por la velocidad característica de la escala $V_{0}$ y

$$
R o=\frac{V_{0}}{\Omega L}
$$

Este último llamado el Número de Rossby, el cual mide la importancia de la fuerza de inercia con respecto a la fuerza de Coriolis.

Para un viento típico de $40 \mathrm{~km} / \mathrm{h}$ y un número de Rossby de orden uno, la fuerza de Coriolis debe de actuar sobre una distancia característica L de casi $150 \mathrm{~km}$. Conforme se aplica en distancias más y más grandes, el efecto de Coriolis se hace mucho más importante.

Tomemos como ejemplo la atmósfera terrestre, el espesor de su capa límite varía entre $200 \mathrm{~m}$ y $1 \mathrm{~km}^{10}$; fuera de esta región, en la capa libre y en condiciones normales, la fricción se puede despreciar. En la capa libre, sin los efectos de Coriolis, el término de aceleración en la ecuación de cantidad de movimiento se balancea por la fuerza debida a la diferencia de presiones y el flujo tiende a estar en dirección del gradiente de presión (de la presión alta hacia la baja).

Sin embargo, en el sistema de viento más simple posible, el del flujo uniforme recto, no hay aceleración y la fuerza debido a la diferencia de presiones se balancea con la fuerza de Coriolis. De esta forma el vector de velocidad se encuentra en ángulos rectos con respecto a la dirección del gradiente de presión. Por lo tanto, las líneas de corriente son casi paralelas a las isóbaras; a esto se le llama Balance Geotrópico.

Es por ello que en la atmósfera, la presión de un flujo uniforme siempre es mayor hacia la derecha (si miramos aguas abajo). El balance Geotrópico se aproxima a las condiciones que se

10 Definiremos lo que es la capa límite planetaria en el siguiente acápite. 
encuentran a unos cientos de metros o más sobre la superficie terrestre. Excepto en caso de vientos ligeros, la magnitud y dirección de los vientos reales a estas alturas, en general, no varían por mas de $10^{\circ}$ y $20 \%$, respectivamente, de sus valores geotrópicos. Es por esto que los mapas isobáricos pueden utilizarse para determinar la velocidad y dirección de los vientos. Las isóbaras conllevan dirección y la magnitud del gradiente (la distancia entre las isóbaras, que también son líneas de corriente), determinan la velocidad.

Cuando el viento sigue una trayectoria curva, la aceleración centrípeta se debe tomar en consideración. Este efecto, el de la aceleración centrípeta, solo es importante cerca del centro de las regiones de baja o alta presión donde las isóbaras tienen curvaturas considerables. Por esto, el viento se desvía de su valor geotrópico y se llama viento de gradiente.

Dentro de la capa límite terrestre, o sea a una altura menor a algunos cientos de metros, los efectos viscosos se vuelven importantes. En particular, la variación de velocidad horizontal con la altura también produce efectos de Coriolis que varían con la altura y la dirección del viento cerca de la superficie terrestre se desviará de su dirección geotrópica o de gradiente.

\section{Capa límite planetaria}

La naturaleza del terreno, la ubicación y la densidad de los árboles, la ubicación y el tamaño de los lagos, ríos, colinas y edificios afectan en gran medida la distribución de la velocidad en la capa límite planetaria. ${ }^{11}$ El cambio en el espesor completo de la capa límite puede variar de unos cuantos cientos de metros hasta varios kilómetros y es más grande en condiciones inestables (grandes urbes con fábricas) en condiciones estables (zona rural).

11 Tomado de Wark y Davis (1998).

760 Ciencia y Sociedad 2013; 38(4): 743-792 
Figura $\mathrm{N}^{\circ} 5$

Efecto de la rugosidad del terreno sobre el perfil de velocidad de la capa límite planetaria

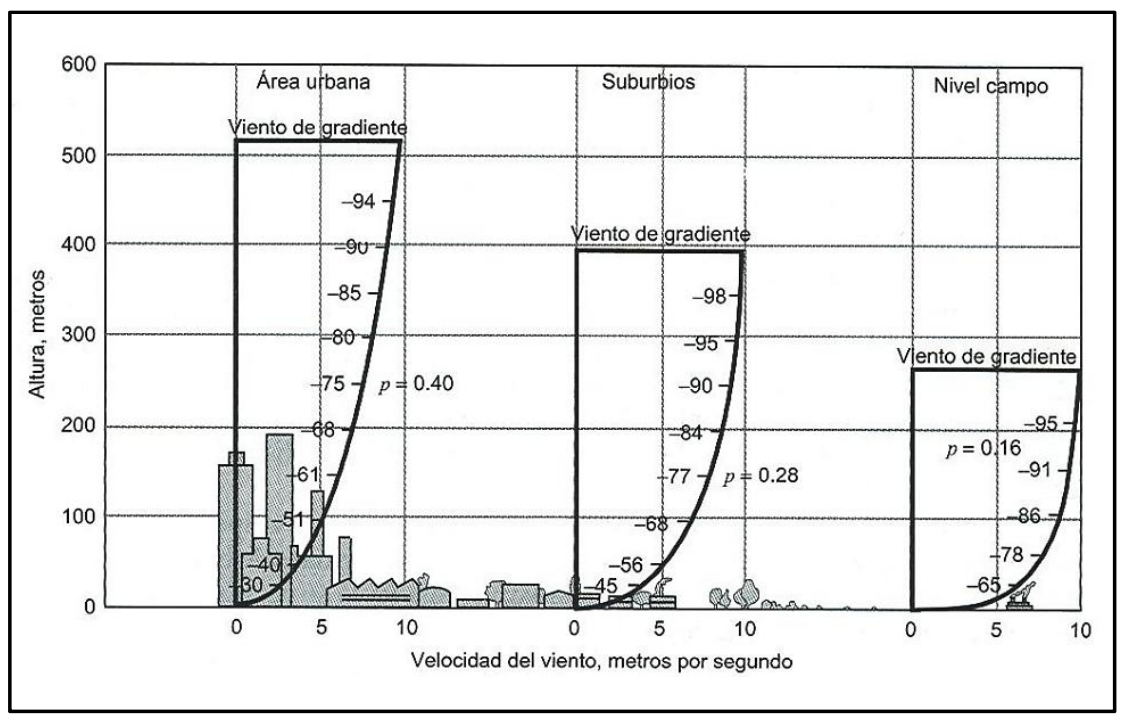

Fuente: Wark y Davis (1998).

La figura N. ${ }^{\circ} 5$ muestra el efecto general de rugosidad del terreno en el perfil, de la velocidad del viento. En este ejemplo, el cambio en el espesor completo de la capa límite va de unos $500 \mathrm{~m}$ hasta los $280 \mathrm{~m}$, para rugosidad decreciente. Debido a los cambios de velocidad con la altura, cualquier valor de velocidad del viento debe citarse en relación con la altura a la que se mide.

La altura estándar internacional para mediciones de la velocidad del viento superficial es de $10 \mathrm{~m}$. A menudo el perfil se modela como una ley de potencias para un flujo en capa límite turbulento sobre una placa plana. Cuando el descenso es casi adiabático y el terreno es nivelado y con una pequeña rugosidad, el exponente $p$ es más o menos 0.15 , que es cercano al valor de $1 / 7$ de la usada para la capa límite turbulenta sobre una placa plana, pero que puede variar con amplitud dependiendo de la rugosidad del terreno y de la estabilidad de la atmósfera. 
Las variaciones en el perfil del viento son importantes por varias razones, pero se observan de manera más directa cuando un avión se aproxima a la pista de aterrizaje. La velocidad del avión con respecto a la velocidad del viento determina su desempeño en la sustentación y el arrastre, pero la velocidad en relación con el suelo determina su patrón de aterrizaje. La velocidad del viento, como es natural, disminuye con la altura debido a la presencia de la capa límite planetaria; el piloto deberá tenerlo en cuenta. La variación rápida en el perfil del viento cerca del suelo se suma a la dificultad en la aproximación.

\section{Intensidad y dirección prevalecientes del viento}

Los patrones circulatorios a gran escala de la atmósfera se modifican por las condiciones locales que, a su vez, pueden variar con el tiempo. Por ejemplo, en las latitudes medias en la capa libre de la atmósfera, las corrientes de aire se curvan y forman las corrientes zonales que son un tipo de movimiento ondulatorio.

\section{Figura N. ${ }^{\circ} 6$}

Rosa de los vientos influenciada por las la intensidad y dirección de los vientos y por el efecto de Coriolis

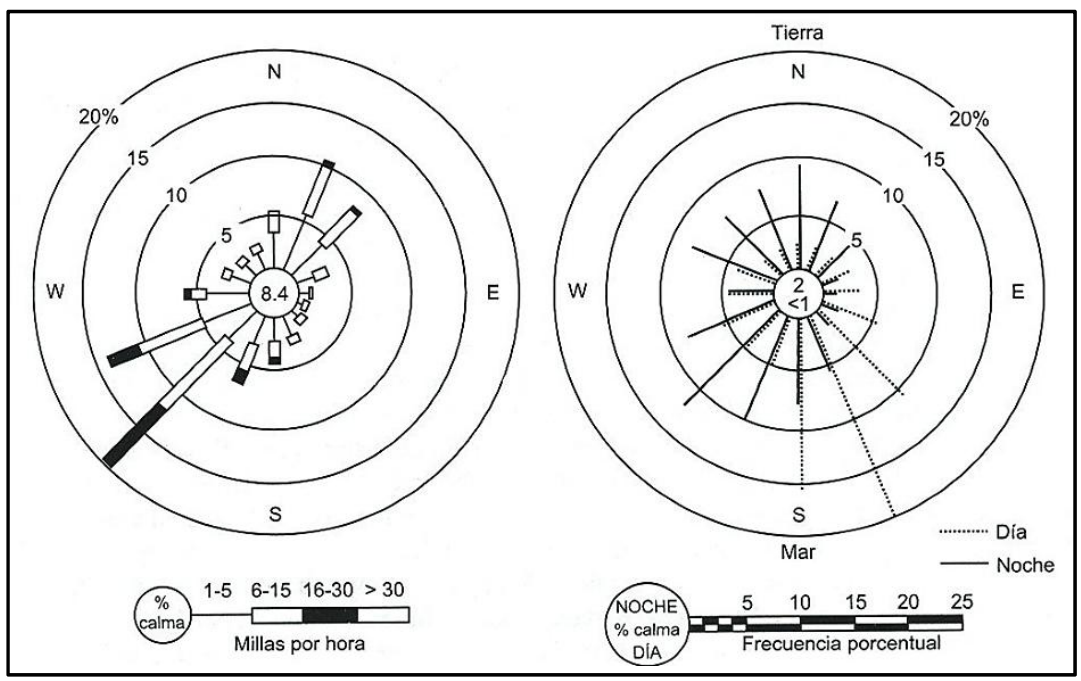


No obstante, hay patrones recurrentes que ayudan a establecer las condiciones estándar en cualquier posición.

Para cualquier tiempo y estación existen expectativas de las condiciones promedio, incluyendo temperatura, lluvia e intensidad y dirección prevalecientes del viento.

\subsection{ESPIRAL DE EKMAN ${ }^{12}$}

El espiral de Ekman es un modelo teórico que explica el movimiento de las capas de un fluido por la acción del efecto de Coriolis. De cómo un viento constante causa movimientos en el agua cerca de la superficie del océano de profundidad ilimitada y viscosidad medida y uniforme, y de cómo el movimiento de una capa de agua afecta a las capas de más abajo.

En el hemisferio norte, las aguas superficiales se moverán en un ángulo de $45^{\circ}$ a la derecha de la dirección del viento y el agua conforme aumenta su profundidad, se movería con direcciones cada vez más hacia la derecha, hasta alcanzar un estado denominado profundidad de Ekman, en la que el agua se movería en una dirección opuesta a la del viento.

La velocidad del flujo de agua es máxima en su superficie y disminuye igualmente con la profundidad. Los vectores de esos movimientos (velocidad de flujo con la orientación que corresponda a cada profundidad) configuran una espiral que es la espiral de Ekman.

12 El primero que notó la desviación de las corrientes superficiales fue el oceanógrafo noruego Fridtjof Nansen durante una expedición al Océano Ártico, a bordo del Fram, donde observó que el desplazamiento de los icebergs no seguía la dirección del viento. En efecto, los icebergs se desplazaban $45^{\circ}$ a la derecha. Al regreso de la expedición en 1886, compartió sus conocimientos y observaciones con el oceanógrafo sueco Vang Walfrid Ekman, quien diez años más tarde publicaría este modelo de circulación llamado espiral de Ekman. 


\section{Figura N. ${ }^{\circ} 7$}

Efecto de la espiral de Ekman

Léase: 1-Viento, 2-Fuerza aplicada desde arriba, 3-Dirección efectiva del flujo de corriente y 4-Efecto Coriolis

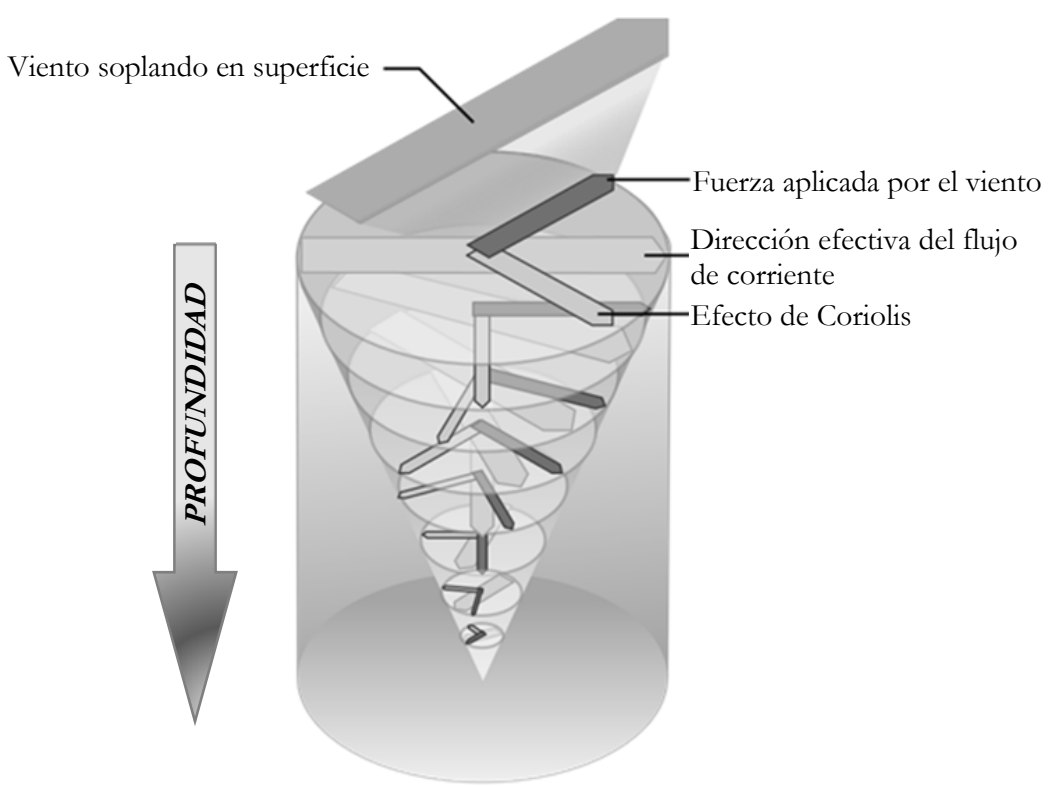

La profundidad de la espiral de Ekman varía con la latitud, pero llega hasta los 100 metros en latitudes medias. En el hemisferio Norte el transporte neto de agua (la resultante de la Espiral Ekman) se produce a $90^{\circ}$ a la derecha de la dirección del viento y es denominado transporte Ekman.

Cuando el transporte de Ekman ocurre cerca de una costa, puede crear una corriente ascendente de agua rica en alimento proveniente de las capas más profundas del océano.

Esto crea una región que es muy buena para la vida del océano. ${ }^{13}$

13 Diccionario de Ciencias de la Tierra (2000). 


\section{Circulación eólica}

La circulación eólica, de una forma u otra forma es consecuencia de la energía o radiación solar. La radiación solar produce la circulación atmosférica que a su vez produce la circulación en los océanos, como ya se explicó con la Espiral de Ekman.

Existen dos tipos de circulación oceánica: la termohalina (cambio de densidad) y la eólica (cambio climático). Para nuestro estudio nos basaremos exclusivamente en la circulación eólica, ya que en el Caribe los cambios de densidad en el viento y océano son mínimos.

Cuando combinamos el principio de Coriolis y la espiral de Ekman, estamos en lo que podría llamarse una circulación horizontal de los océanos y los vientos.

Básicamente el viento al soplar sobre la superficie mueve el agua por fricción, pero debido al efecto de la rotación de la tierra, Fuerza de Coriolis, el transporte neto es realmente a la derecha (o $90^{\circ}$ ) de la dirección del viento.

\section{Figura N. ${ }^{\circ} 8$}

Relación de la circulación de los vientos y las corrientes superficiales que conjuntamente con la fuerza de Coriolis forman la Espiral de Ekman
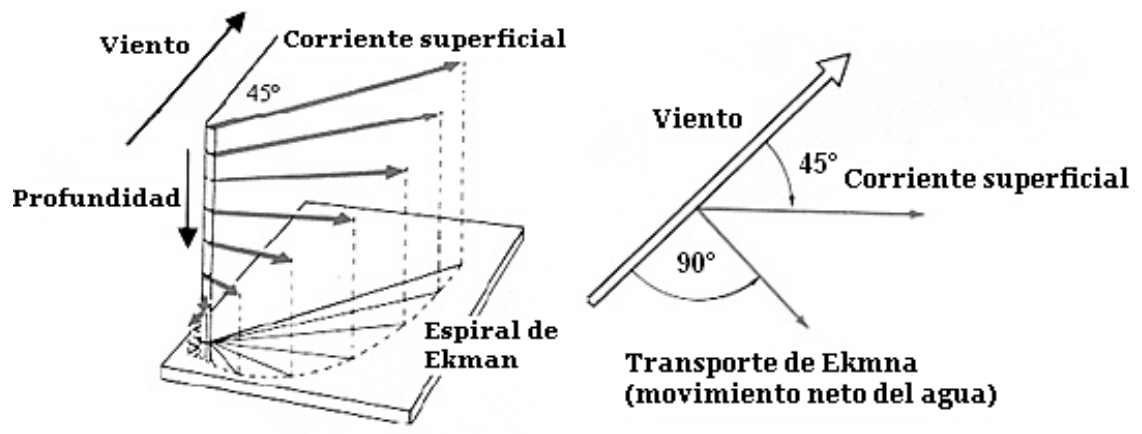
Nótese en la figura cómo el agua de la superficie se mueve exactamente a $45^{\circ}$ respecto de la dirección del viento debido a la fuerza de Coriolis. Como existe fricción entre las diferentes capas de agua en el océano, a medida que se aumenta la profundidad, cada capa tiende a tener menor velocidad y el equilibrio de las fuerzas: fricción entre el viento y el agua, fricción entre las capas de agua y la fuerza de Coriolis, hace que cada una de ella rote más a la derecha con respecto a la capa de arriba, produciéndose, de esta manera, una espiral, conocida como Espiral de Ekman. El océano podrá rotar hasta $180^{\circ}$ respecto de la dirección en superficie, pero en promedio -se dice- que el transporte es a $90^{\circ}$ respecto de la dirección del viento.

Sin embargo, también hay que tener en cuenta hasta cierto punto la circulación termohalina. El término se refiere a la circulación o movimientos del agua cuando hay cambios de densidad producidos ya sea por cambios en temperatura y salinidad.

\section{Figura N. ${ }^{\circ} 9$}

Esquema de la formación de una corriente geostrófica.

Evolución y comportamiento de la espiral

y capa Ekman en superficies de flujos

Atlantico Norte

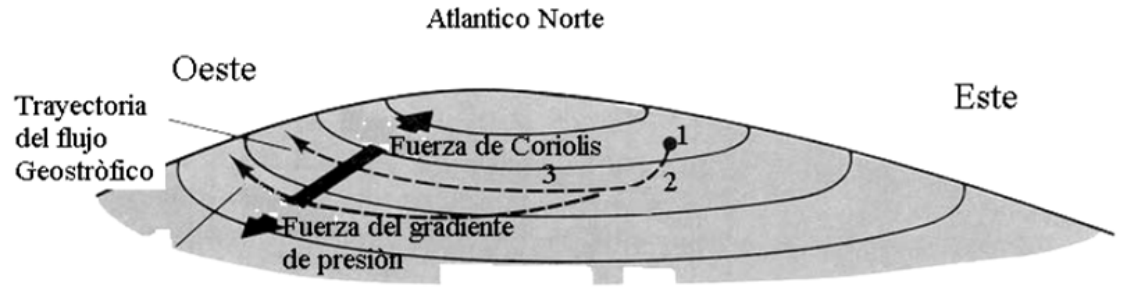

El calor en el océano es distinto a calentar agua en un "beaker" por debajo, donde el agua ascendería. El océano es calentado por encima. Sabemos que si un extremo de un tanque de agua se calienta desde la superficie, esta agua se expande y, por lo tanto, sube su nivel. Esta diferencia de nivel con respecto al lado frío hace que fluya agua desde la caliente a la fría (aunque no es una 
circulación en sentido estricto). Así en nuestro globo terráqueo es conocido el mayor calentamiento en las latitudes bajas y por lo tanto hay un flujo hacia los polos. Aunque esto ocurre, no es el único responsable en la formación de corrientes.

Podemos notar cómo un desnivel en la superficie del agua produce un movimiento (etapa 1) en la dirección gradiente de presión, o sea, de lo más alto a lo más bajo. Pero debido a la rotación de la tierra, una vez que se inicia el movimiento, la fuerza de Coriolis modifica su dirección desviándola hacia la derecha (etapa 2) en el Hemisferio Norte (izquierda en el Hemisferio Sur), hasta que la fuerza del gradiente de presión (P) y la fuerza de Coriolis (C) lleguen a equilibrarse. Esto lo logra cuando ambos están en dirección opuesta (etapa 3), haciendo que la dirección del fluido sea siempre perpendicular a ambas fuerzas, estando siempre a la derecha de ella la fuerza de Coriolis (C) y en la izquierda la fuerza del gradiente de presión (P) en el Hemisferio Norte (contrario en el Hemisferio Sur). Este es el origen de la mayoría de grandes corrientes en el Océano, entre ellas muy conocidas: Corriente del Golfo, Corriente de Kuroshio y que son llamadas corrientes geostróficas (y que son termohalina en su origen). ${ }^{14}$

\section{Circulación y masa de agua}

Para hablar de ello debe dividirse el mundo (o los océanos) por partes. Por ejemplo: Atlántico Ecuatorial del Norte o Atlántico Ecuatorial del Sur, y sus mares adyacentes, como el Mar Mediterráneo o el Mar de Labrador. Pero debe tenerse en cuenta, que cada división que se haga es influenciada por los mares de afuera.

Cuando se estudia circulación y masas de agua (estudio del carácter del agua: temperatura $(\mathrm{T})$, salinidad $(\mathrm{S})$, densidad $(\alpha)$, oxígeno disuelto $\left(\mathrm{O}_{2}\right)$, contenido de nitrato $\left(\mathrm{NO}_{3}\right)$, etc., no se sabe qué se

14 Evolución y comportamiento de la espiral y capa Ekman en superficies de flujos.

Fuente: Pinallai y Villanueva (2012). 
estudia primero. Es igual que la cuestión de quién fue primero, el huevo o la gallina. En realidad, ¿qué es lo que llamamos capa superficial? Se conoce como tal la capa encima de la cual el decrecimiento de $\mathrm{T}$ con profundidad es pequeño.

Puede ser entre 300 y 1000 metros. Es una capa muy estable. La capa profunda es menos estable. Mientras que la capa en contacto con el fondo tiene propiedades distintas y es llamada "agua de fondo".

La circulación oceánica responde al patrón general de la circulación atmosférica. El viento sobre la superficie del océano es realmente el motor de las grandes corrientes oceánicas superficiales que conocemos. Este patrón de viento es en promedio permanente, solo se rompe en eventos extraordinarios como el Fenómeno de El Niño, en donde este cambia el patrón de circulación del viento, que a la vez cambia el patrón de circulación del océano. El patrón de viento

Figura N. ${ }^{\circ} 10$ Patrón promedio de la circulación del viento sobre la superficie del planeta

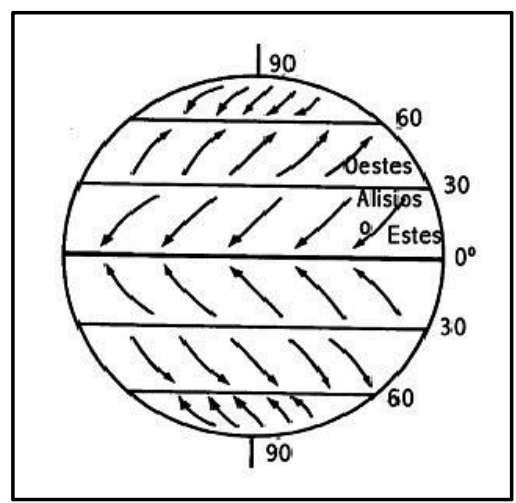
promedio se muestra en la figura a la derecha.

La circulación en los océanos es muy similar, solo se diferencian en detalles. En general, hay circulación a favor de las manecillas del reloj en el Atlántico y Pacífico Norte, y una circulación contra reloj en el sur (Atlántico, Pacífico e Índico).

En el Pacífico y Atlántico hay una intensificación de las corrientes sobre los lados oeste de los océanos (Corriente del Golfo y Corriente de Kuroshio). Hay alguna evidencia de intensificación en el Atlántico Sur y Océano Índico, pero al oeste del Pacífico Sur, la circulación es compleja y no es claro que se intensifique. 
En el Ecuador los tres océanos tienen una corriente fluyendo hacia el oeste Ecuador: la Corriente Ecuatorial del Sur, y también hacia el oeste al norte del Ecuador, la Corriente Ecuatorial del Norte. En los tres océanos estos dos flujos hacia el oeste están separados por un flujo hacia el este: la contracorriente Ecuatorial. Estas contracorrientes se derivan de las variaciones de fricción del viento desde el norte y sur del Ecuador, en la Zona de Confluencia Intertropical.

Otra característica del sistema ecuatorial es la Corriente Sub-superficial que fluye hacia el este, cerca de 100 metros de profundidad, producida por el acumulamiento de aguas en el oeste de los océanos por el viento en la superficie (vientos alisios). La circulación en las aguas profundas es un flujo hacia el norte y sur, y más intenso sobre los lados oestes, como se nota en la gráfica del modelo de circulación de Stommel. ${ }^{15}$

La más intensa es la Corriente del Golfo, corriente que al salir frente al Cabo Hatteras sobre la costa Este de los Estados Unidos, muestra un serpenteo al internarse en el Océano Atlántico como muestra la figura N. ${ }^{\circ} 11$. De estos serpenteos se producen remolinos que se desprenden de la corriente con aguas frías (color celeste) atrapadas desde el norte, de la Corriente de Labrador, como muestra la imagen infrarroja de

Figura N. ${ }^{\circ} 11$ Circulación de Stommel

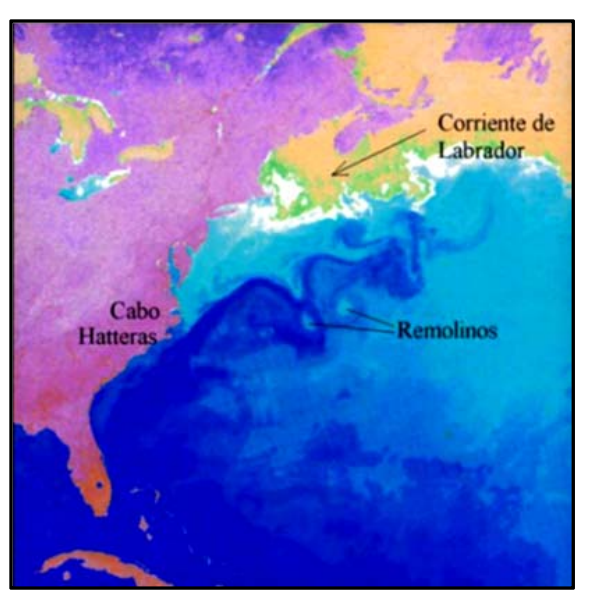
la figura.

15 Modelo de Circulación de Stommel, Bulgakov y Skiba (11 de Septiembre de 2003). Centro de Ciencia de la Atmósfera, UNAM. 
Por todo lo anterior es evidente que el patrón de circulación ecuatorial que se deduce del patrón de los vientos promedio.

Es por esto que algunas de las especies marinas que encontramos en la Isla Galápagos en el Ecuador o del Coco de Costa Rica tienen origen en el Indo-Pacífico. Solo a través de una corriente que viaje de oeste a este podría explicarse la existencia de estas especies en nuestras costas. Es indudable que la existencia de la Contracorriente Ecuatorial que viaja de oeste a este y que en el mes de octubre es claramente intensificada.

\section{Consecuencia fatal: las islas de basura}

Desde la época de los viajes de Colón, América se reconoce por la existencia del Mar de los Sargazos. El mar de los Sargazos es una región del océano Atlántico septentrional que se extiende entre los meridianos $70^{\circ}$ y $40^{\circ} \mathrm{O}$ y los paralelos $25^{\circ}$ a $35^{\circ} \mathrm{N}$, y que en los siglos XVII al XVIII tuvo la tétrica fama de ser lugar de cementerio de buques de navegación a vela. ${ }^{16}$ El sargazo es un tipo de alga que generalmente crece adherida a las rocas cercanas a la costa, pero se ha adaptado por completo a la vida pelágica y ahora flota en las capas superiores del océano. Dos factores le permiten al sargazo permanecer en la zona y dominarla: las corrientes lentas que circulan alrededor y mantienen en su interior la mayor parte de las algas y su reproducción por fragmentación, lo que permite que cada pequeño fragmento que se desprende dé lugar a una planta nueva.

Es el mar de los Sargazos la primera isla de basura, que conoció el mundo y que ahora sirve de base para la conformación de una de las islas de basuras más grandes del mundo.

16 http://www.ecured.cu/index.php/Mar_de_los_Sargazos 
Figura N. ${ }^{\circ} 12$

El plástico se acumula en enormes islas de basura en los océanos

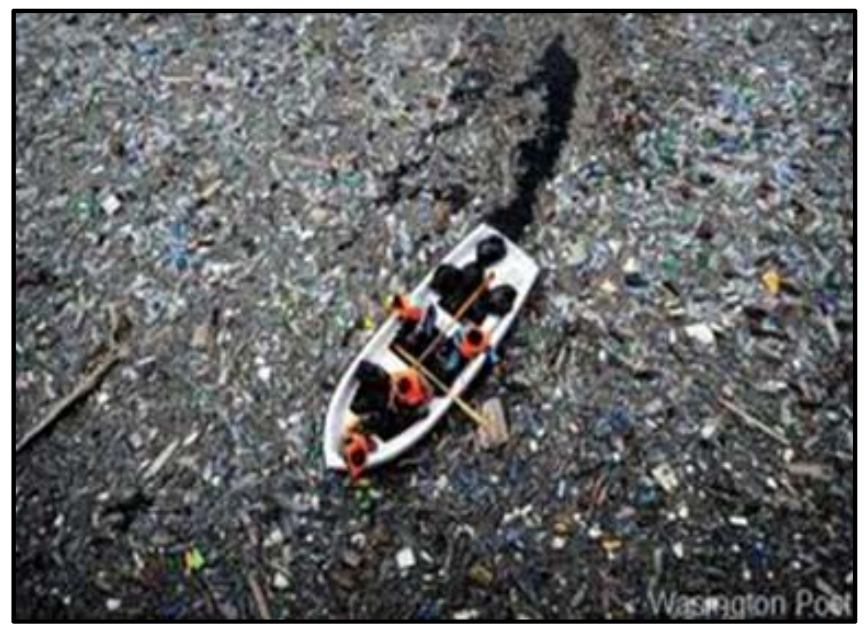

Desde el año de 1986 investigadores estadounidenses del Woods Hole Oceanographic Institution y de la Universidad de Hawái han estado estudiando el cómo y por qué se han formado las islas de basura en los océanos Atlántico y en el Pacífico, lo que está por explicarse aún son las consecuencias que tendría en los seres vivos semejante contaminación.

Entre 1986 y 2008 se han recolectado muestras por más de 7,000 estudiantes en 6,136 localidades del Mar Caribe, del Océano Atlántico y el Pacífico.

Solo para la isla formada en el Atlántico Norte los investigadores determinaron que los residuos flotantes tardan menos de 60 días en llegar desde las costas norteamericanas hasta la zona de acumulación, la gigantesca isla de plástico se formó con desechos arrojados desde las tres Américas.

No obstante, el mayor caudal proviene del norte. Fueron arrastrados por corrientes superficiales y, por una circulación ciclónica de vientos, se aglutinaron en una zona de convergencia, en el mar 
de los Sargazos. Se trata de "una especie de embudo, donde el agua se concentra y termina yéndose hacia el fondo", según José Luis Esteves, esto es lo que habíamos demostrado a consecuencia del Principio de Coriolis y la Espiral de Ekman.

\section{Figura N. ${ }^{\circ} 13$ \\ Posición y composición energética de la isla de basura del Atlántico Norte}

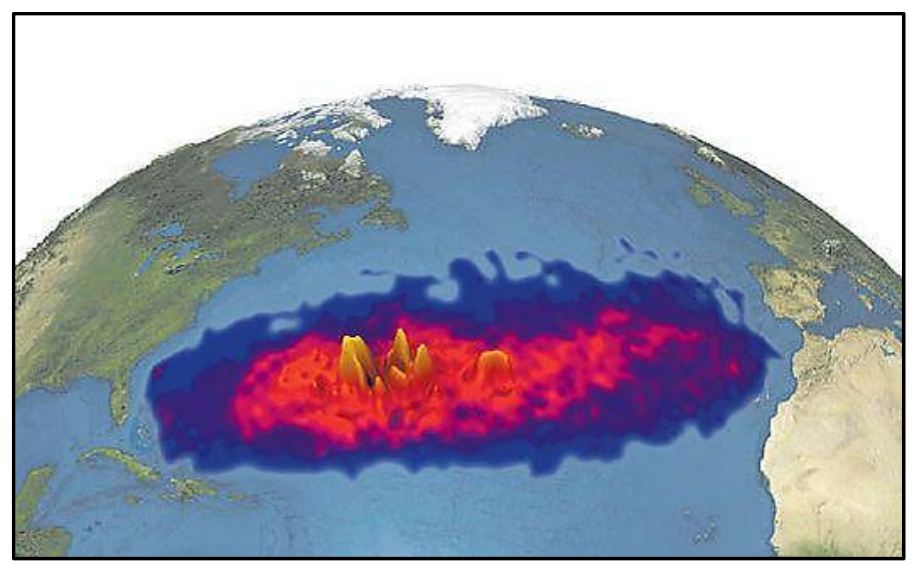

Estas islas de basura de las cuales estamos hablando, como consecuencia del uso irracional que hacemos los humanos, están formadas fundamentalmente por diversos tipos de plásticos. La mayor de estas se encuentra ubicada en el Océano Pacífico, dos en el Atlántico (una en el Atlántica Norte-Mar de los Sargazos-, y otro en el Atlántico Sur), una en el Océano Índico, y dos más pequeñas: una en el Mar Mediterráneo y la otra en el Mar Caribe.

Dos definiciones que nos van a ayudar en la explicación de estos fenómenos son: ${ }^{17}$

17 Recuperado de http://www.google.com.do/imgres?imgurl=http://www.voyages photosmanu.com/Complet/images/corrientes_oceanicas.gif\&imgrefurl=http://

www.voyagesphotosmanu.com/corrientes_oceanicas.html\&h $=335 \& w=619 \& s z=15$ \&tbnid= $=$ MLHO3aLOsBCsM:\&tbnh $=66 \&$ tbnw $=122 \& z o o m=1 \& u s g=$ OJjA0d59DXCEz-1ISyIk=\&docid=4_2LM27t-yP8AM\&sa=X\&ei=4p7RUezIIOni 4AOS9oG4BA\&sqi=2\&ved=0CE4Q9QEwBQ\&dur=198 


\section{Corriente oceánica o marina:}

Es un movimiento superficial de las aguas de los océanos y en menor grado, de los mares más extensos. Estas corrientes tienen multitud de causas, principalmente, el movimiento de rotación terrestre (que actúa de manera distinta y hasta opuesta en el fondo del océano y en la superficie) y por los vientos constantes o planetarios, así como por la configuración de las costas y la ubicación de los continentes.

Figura N. ${ }^{\circ} 14$

\section{Corrientes marinas del mundo}

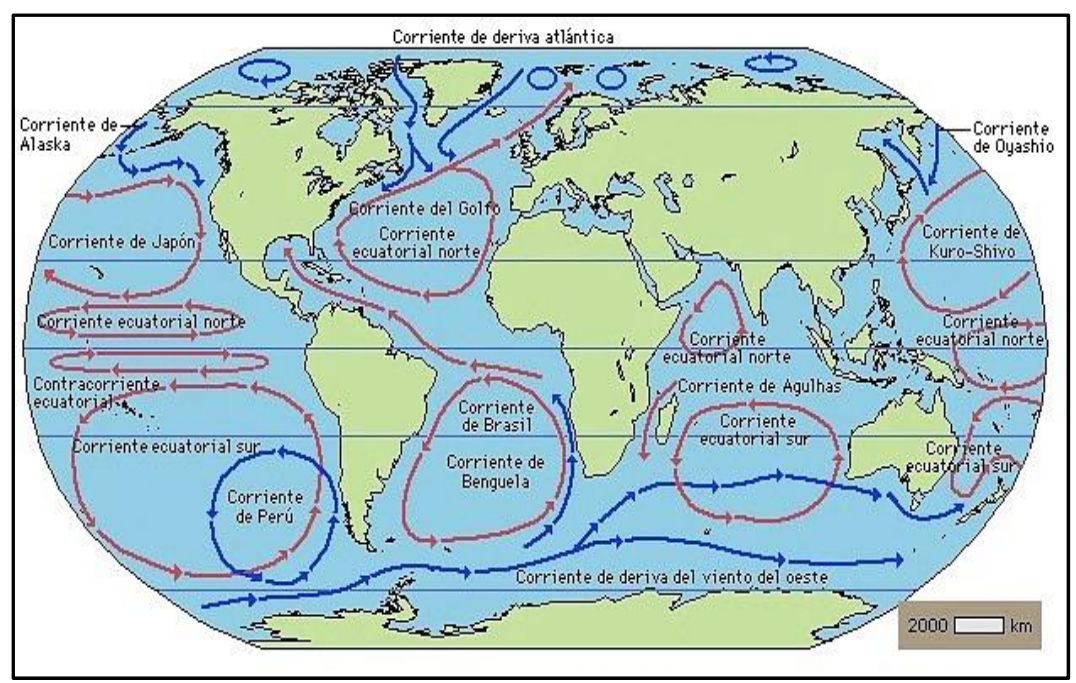

Suele quedar sobreentendido que el concepto de corrientes marinas se refiere a las corrientes de agua en la superficie de los océanos y mares (como puede verse en el mapa de corrientes) mientras que las corrientes submarinas no son sino movimientos de compensación de las corrientes superficiales. Esto significa que si en la superficie las aguas superficiales van de este a oeste en la zona intertropical (por inercia debido al movimiento de rotación terrestre, que es de oeste a este), en el fondo del océano, las aguas se desplazarán siguiendo ese movimiento de rotación de oeste a este. 
Giro Oceánico: o simplemente giro, en oceanografía es cualquier gran sistema de corrientes marinas rotativas, particularmente las que están relacionadas con los grandes movimientos del viento. Los giros son causados por el efecto de Coriolis, por la vorticidad planetaria junto con la fricción horizontal y vertical, determinan los patrones circulatorios del viento (momento de fuerza).

Figura N. ${ }^{\circ} 15$

\section{Giros oceánicos}

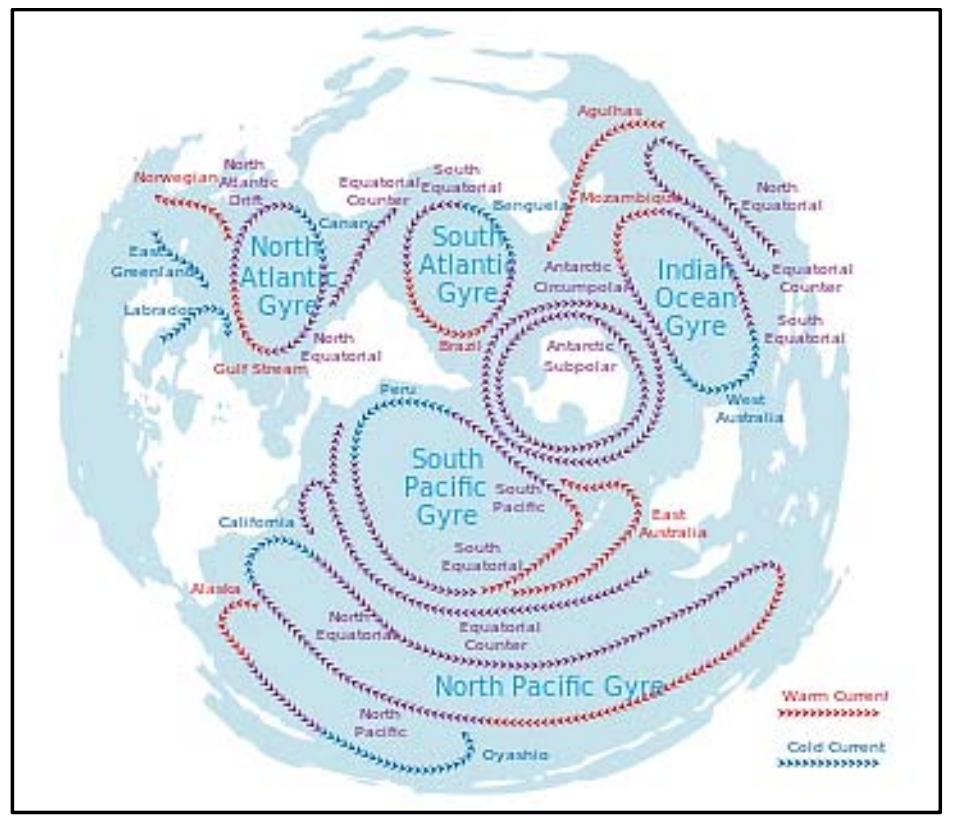

Los siguientes cinco giros son los más notables: ${ }^{18}$

- Giro del Atlántico Norte;

- Giro del Atlántico Sur;

- Giro del Océano Índico;

- Giro del Pacífico Norte;

- Giro del Pacífico Sur.

18 http://es.wikipedia.org/wiki/Giro_oce\%CC3\%A1 nico 
Coincidiendo con los giros oceánicos y a consecuencia de las corrientes marinas, están ubicadas las diferentes islas de basura que existen en nuestros océanos.

La isla de basura del Océano Pacífico Norte se extiende desde las coordenadas $135^{\circ}$ a $155^{\circ} \mathrm{O}$ y $35^{\circ}$ a $42^{\circ} \mathrm{N}$ con un tamaño aproximado de entre 700,000 y 1,400,000 km². La que se encuentra en el Océano Atlántico Norte se sitúa entre el paralelo $22^{\circ} \mathrm{N}$ y $38^{\circ} \mathrm{N}$ con unos $4.000 .000 \mathrm{~km}^{2}$.

No existen muchos estudios sobre el Mar mediterráneo, pero se ha detectado cerca de la isla de Elba una acumulación de plásticos con una extensión cercana a $900 \mathrm{~km}^{2}$, según investigadores de la Universidad de Lieja, y se calcula que se encuentran a la deriva unos 3,500,000 de Tm de plástico en total. Estas islas no aparecen en las imágenes de satélite, por lo que es difícil evaluar su extensión y estudiar su dinámica.

Para la isla de basura existente entre la península del Yucatán y la Isla de la Juventud, no se tiene casi información y solo se ha mencionado en uno u otro artículo periodístico sin mucha base científica, pero es un hecho irrefutable de que existe la misma.

De acuerdo al SEA ${ }^{19}$, los desperdicios concentrados en las diferentes islas de basura provocan un remolino (Espiral de Ekman) provocado por las corrientes marinas y la circulación eólica, esto impide que se dispersen hacia la costa y hace que las partículas más pequeñas y finas se vayan hacia el fondo.

Diversos investigadores que trabajan en diferentes áreas oceánicas han encontrado concentraciones de plástico cercanas a un millón de partes por milla cuadrada; en muchos casos la concentración hallada era siete veces mayor que la concentración de zooplancton en la zona. Muestras tomadas de aguas más profundas indican

19 SEA: Shepherd Conservation Society. 
que los niveles disminuyen con la profundidad por lo que la mayor concentración se da en la superficie.

Los científicos norteamericanos, Curtis Ebbesmeyer y James Ingraham pudieron ajustar un modelo teórico en 1988 que puede predecir el comportamiento de las islas de basura, la cual fue descubierta por Charles Moore en 1997. Quince años después de ese avistamiento, esa porción de desechos flotantes no es un espejismo. Está creciendo, impulsada, según Greenpeace, por las 6 millones de toneladas de residuos que caen al mar cada año. De hecho, el Instituto Scripps de Oceanografía, en EE. UU., indicó que esa "sopa de plástico" en alta mar se ha multiplicado por 100 entre 1999 y el 2010.

\begin{tabular}{|c|c|c|c|c|c|}
\hline \multicolumn{7}{|c|}{ Comparación Tamaño (masa de agua) } \\
\hline $\begin{array}{c}\text { Basura } \\
\mathbf{k m}^{\mathbf{2}}\end{array}$ & $\begin{array}{c}\text { Pacífico } \\
\mathbf{k m}^{\mathbf{2}}\end{array}$ & $\begin{array}{c}\text { Atlántico } \\
\mathbf{k m}^{\mathbf{2}}\end{array}$ & $\begin{array}{c}\text { Índico } \\
\mathbf{~ k m}^{\mathbf{2}}\end{array}$ & $\begin{array}{c}\text { Antártico } \\
\mathbf{k m}^{\mathbf{2}}\end{array}$ & $\begin{array}{c}\text { Ártico } \\
\mathbf{k m}^{\mathbf{2}}\end{array}$ \\
\hline $3,400,000$ & $155,557,000$ & $76,762,000$ & $68,556,000$ & $20,327,000$ & $14,056,000$ \\
\hline
\end{tabular}

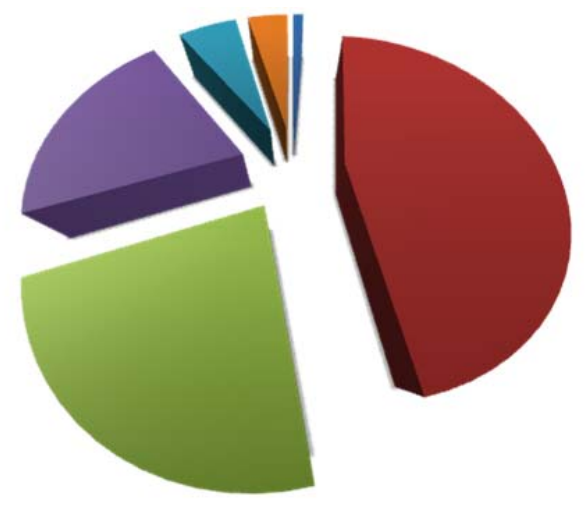

Basura km²

Pacífico $\mathrm{km}^{2}$

Atlántico km²

I Índico km²

Antártico km²

Ártico km² 


\section{La ubicación de las Islas de Basura}

La mayor de las islas, la del pacífico, está situada a unos 1.000 kilómetros de Hawái; este vertedero marítimo tiene unas dimensiones increíbles. Se calcula que ocupa de 1,7 millones a 3,4 millones de kilómetros cuadrados, y pesa unos 3,5 millones de toneladas. Argentina tiene una superficie de casi 2,8 millones de kilómetros cuadrados. Una catástrofe ecológica que, por desgracia, no deja de crecer. (Esto fue publicado el 22 de mayo de 2012). Los expertos calculan que tiene una superficie de 3.4 millones de kilómetros cuadrados. Más de una vez la superficie de Argentina (2,8 millones $\left.\mathrm{km}^{2}\right)^{20}$ y más de 70 veces la de República Dominicana $\left(48,442 \mathrm{~km}^{2}\right)$.

\begin{tabular}{|c|c|c|}
\hline \multicolumn{2}{|c|}{ Comparación tamaño (masa continental) } \\
\hline $\begin{array}{c}\text { Isla de } \\
\text { Basura km } \mathbf{~ k}^{\mathbf{2}}\end{array}$ & $\begin{array}{c}\text { Argentina } \\
\mathbf{k m}^{\mathbf{2}}\end{array}$ & $\begin{array}{c}\text { República } \\
\text { Dominicana km }\end{array}$ \\
\hline $3,400,000$ & $2,800,000$ & 48,442 \\
\hline
\end{tabular}

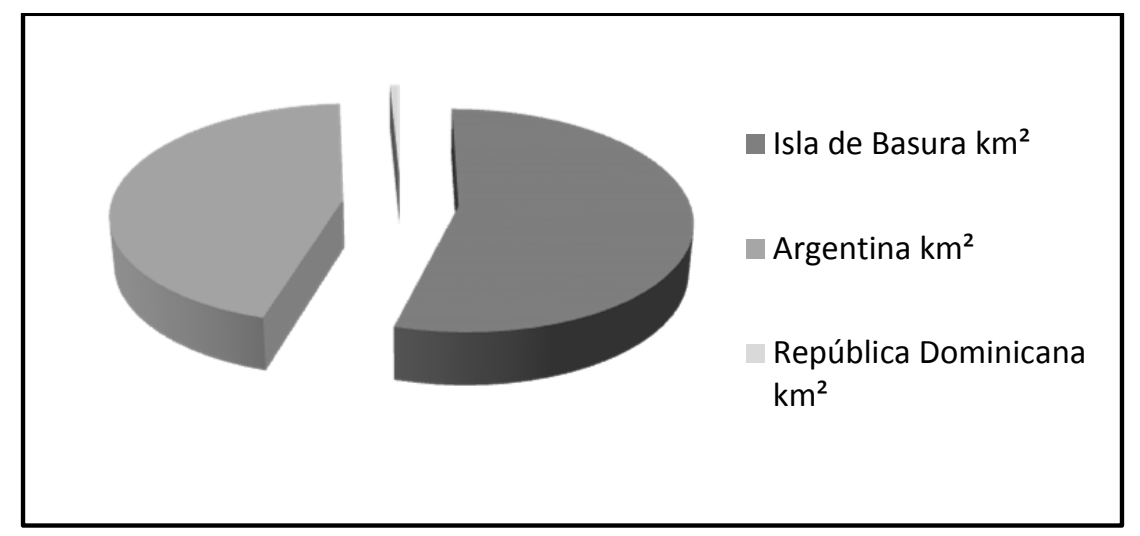

${ }^{20}$ Recuperado de http://blogs.tudiscovery.com/descubre-el-verde/2011/04/con taminaci $\% \mathrm{C} 3 \% \mathrm{~B} 3 n$-de-pl $\% \mathrm{C} 3 \% \mathrm{~A} 1$ stico-en-el-oc $\% \mathrm{C} 3 \% \mathrm{~A} 9$ ano-responsabilidadesproblemas-y-soluciones.html 
El tamaño de la zona afectada se desconoce, aunque se estima que es de $700.000 \mathrm{~km}^{2}$ a más de 15 millones de $\mathrm{km}^{2}(0,41 \%$ a $8,1 \%$ del tamaño del océano Pacífico). El área puede contener cerca de 100 millones de toneladas de desechos. También se ha sugerido que la mancha puede presentar dos zonas de basuras, que están vinculadas.

Lo desconcertante es que la Administración Nacional Oceánica y Atmosférica dice que el $80 \%$ de esos restos lo producen fuentes terrestres y el $20 \%$ restantes, los barcos. Las corrientes portan desechos desde la costa oeste de Norteamérica hacia el vórtice en unos 5 años, y los desechos de las costas este de Asia en un año o menos. La basura de

Figura N. ${ }^{\circ} 16$ Muestra de los estragos causados por las Islas de Basura

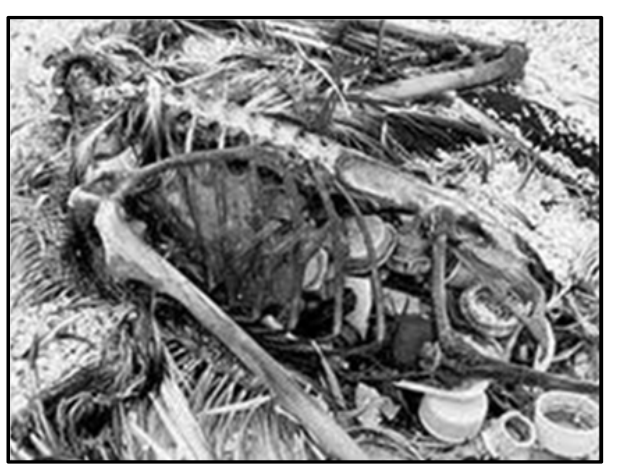

origen terrestre llegaría a partir de cursos de agua dulce contaminados con plásticos que han salido del círculo de reciclaje (aproximadamente se recicla el 5\% del plástico en los países industrializados).

La isla de basura está compuesta por todo lo que se pueda imaginar: boyas, redes de pesca, cepillos de dientes, bombillas, tapas de botellas, objetos procedentes de alcantarillas, etc. Pero destacan sobre todo, pequeñísimas piezas de plástico, millones de ellas, algunas del tamaño de un grano de arroz. El efecto es muy parecido al que ejerce el mar sobre la arena de la playa, pero en su versión más espantosa.

Juan Manuel Díaz, director científico de la Fundación Marviva, que trabaja por la sostenibilidad marina en Costa Rica, Colombia y Panamá, explica que la basura puede llegar allí desde Canadá, 
Asia o América Latina, situación que está dañando la fauna. Miles de ballenas y tortugas quedan atrapadas en redes. Sin contar la intoxicación con químicos de animales que luego son consumidos por el hombre. La revista Marine Ecology Progress publicó un estudio que señala que los peces en el Pacífico ingieren hasta 24 mil toneladas de plástico al año. Además, los desechos son aprovechados por una invasiva araña acuática (Halobates sericeus) como incubadora, especie que ahora abunda y puede llegar a cambiar el ecosistema. Los científicos calculan que en veinte años la mancha que flota en el Pacífico tendrá la extensión de un continente.

Esta crisis sanitaria no es exclusiva del Pacífico. Díaz Merlano señala que sucede algo similar en el mar de los Sargazos, entre la Península de Yucatán y la Isla de la Juventud, donde existe otra "isla de basura", un nuevo giro oceánico similar al del Pacífico y que también se ve en otras zonas del Atlántico. Los residuos son difíciles de detectar si no es con expediciones, pues están ubicados a pocos metros por debajo de la superficie y desintegrados al punto de que parecen plancton, no son localizables vía satélite.

Mancha de basura del Atlántico Norte (North Atlantic Garbage Patch), o "Gran Mancha de basura" plástica en el Atlántico Norte, ${ }^{21}$ es una nueva zona de desechos marinos descubierta flotando en el Giro del Atlántico Norte.

La mancha se estima en cientos de kilómetros de largo 22 , con una densidad de 200.000 fragmentos de basura flotante por kilómetro cuadrado. ${ }^{23}$ La zona de detritos cambia de posición en $1600 \mathrm{~km}$ norte y sur estacionalmente, y se desplaza aún más

\footnotetext{
21 Recuperado de http://blogs.tudiscovery.com/descubre-el-verde/2011/04/conta minaci $\% \mathrm{C} 3 \% \mathrm{~B} 3 \mathrm{n}-\mathrm{de}-\mathrm{pl} \% \mathrm{C} 3 \% \mathrm{~A} 1$ stico-en-el-oc $\% \mathrm{C} 3 \% \mathrm{~A} 9$ ano-responsabilidadesproblemas-y-soluciones.html

22 Recuperado de http://es.wikipedia.org/wiki/Isla_de_basura

23 Recuperado de http://isladebasura.com/
} 
lejos durante el fenómeno climático de El Niño según datos de la Administración Nacional Oceánica y Atmosférica (National Oceanic and Atmospheric Administration, NOAA).

\section{Figura N. ${ }^{\circ} 17$}

Posición de las islas de basura del Atlántico Norte y Mar Caribe

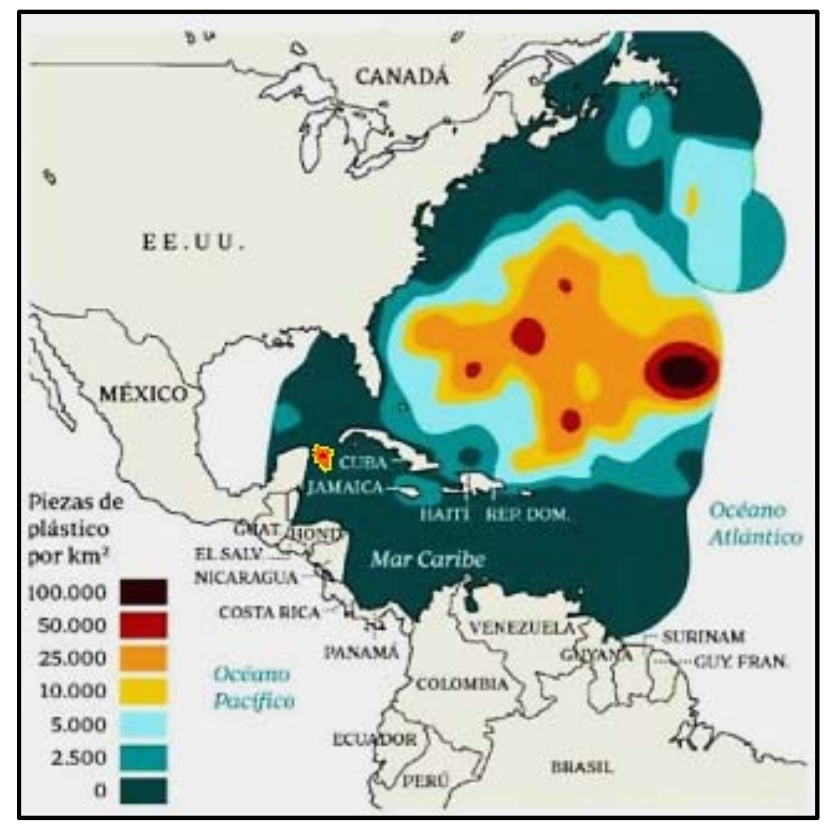

No existen trabajos sobre posibles islas de basura en el mar Mediterráneo, pero no se descarta su existencia. "Las corrientes superficiales son diferentes a las de los océanos, pero hay puntos negros de contaminación perfectamente localizados como el Estrecho de Gibraltar o Algeciras".

\section{La isla de basura del Caribe}

El Mar Caribe tiene la influencia de la corriente ecuatorial del Atlántico Norte, que recorre desde el Cabo de Buena Esperanza (África) hasta la Península de La Florida, EE. UU. A esta corriente se le une la corriente ecuatorial del Atlántico. 


\section{Figura N. ${ }^{\circ} 18$}

\section{Corrientes marinas}

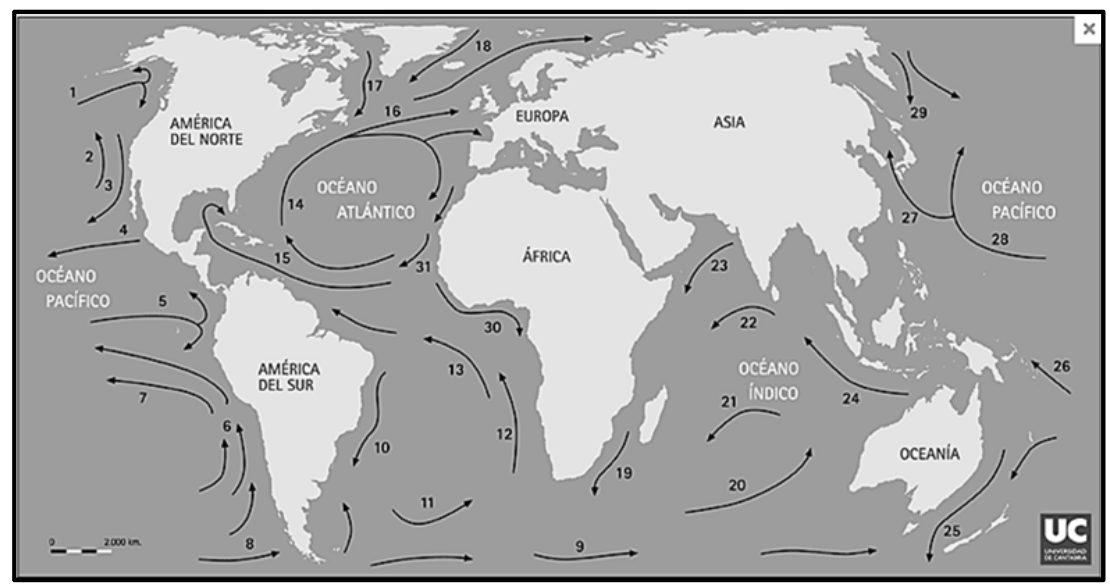

Toda la basura de la costa norte de Brasil y Las Guayanas es recogida por la corriente ecuatorial del Atlántico, la que al unirse con la Ecuatorial del Atlántico Norte, hace suya las basuras de la costa de las Antillas Menores, la costa norte de Venezuela, Colombia, la costa sur de Puerto Rico, República Dominicana, Haití y Jamaica.

La basura que es arrastrada por las corrientes al chocar con la punta de la península de Yucatán al oeste, y de la punta occidental de la Isla de Cuba, forman un remolino (Espiral de Ekman), que da origen a la isla que se nos está formando en el Caribe.

De acuerdo con los autores de este documento, la isla de basura del Caribe está ubicada aproximadamente a los 21 grados de latitud Norte, 65 longitud Oeste. Frente a las costas de Belice, y de la Isla de la Juventud.

El tamaño de la misma varía ya que, por suerte, para los habitantes del Caribe con cada época ciclónica la misma se reduce ya que los vientos y las corrientes marinas producidas por los diversos huracanes que nos azotan cada año; la isla de basura se reduce y envía su carga de basura a la isla del Atlántico Norte. 
Figura N. ${ }^{\circ} 19$

Posición de la isla de Plástico en el Mar Caribe

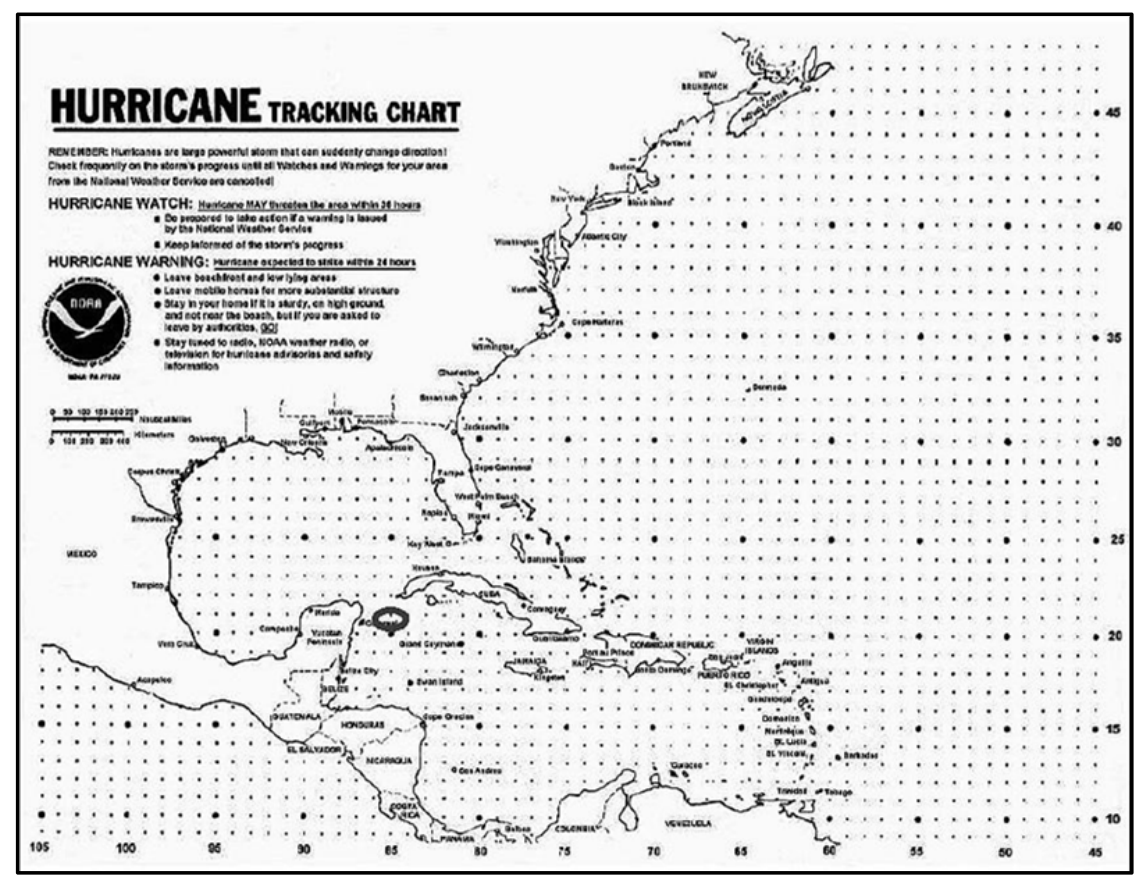

[Nota: La posición en el mapa es criterio y aporte de los autores de este artículo].

Sin embargo, el hecho de tener una isla de basura en esta zona está afectando enormemente a los arrecifes coralinos centroamericanos ubicados frente a las costas de Honduras y Belice.

\section{Consecuencias de las islas de basura: Algunas conclusiones}

\subsection{FotOdEGRADACIÓN DE LOS PLÁSTICOS EN EL OCÉANO}

A diferencia de los desechos biodegradables, los plásticos fotodegradables se desintegran en pedazos más pequeños, aunque permanecen siendo polímeros. Este proceso continúa hasta llegar a nivel molecular. 
Figura N. ${ }^{\circ} 20$

Situación de las barreras coralinas

frente a las costas centroamericanas

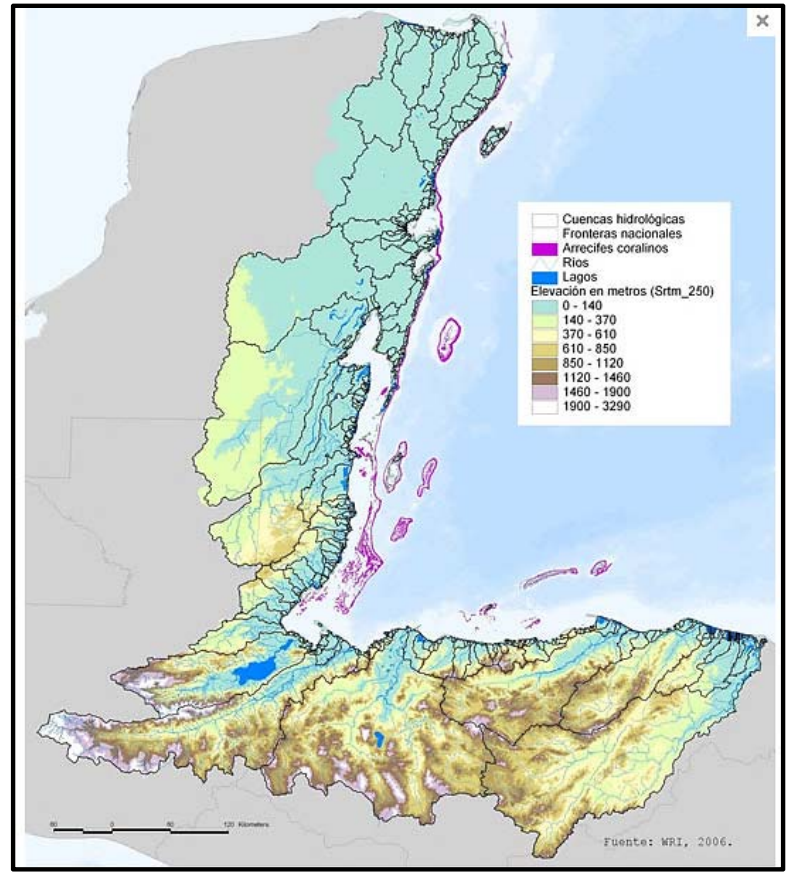

Como los desechos plásticos flotantes fotodegradables se convierten en trozos más pequeños cada vez, se concentran en la parte superior hasta que se desintegran, y el plástico al final llega a ser de un tamaño tan pequeño que puede ser comido por los organismos marinos que viven cerca de la superficie del océano. Por lo tanto, los residuos de basura entran por completo en la cadena alimenticia.

A pesar de la descripción hecha por Charles Moore, la mancha de basura del este no se caracteriza por ser una zona visible de desechos flotantes. El proceso de desintegración significa que las partículas de plástico más peligrosas son demasiado pequeñas como para ser vistas. Los investigadores estiman la densidad total de la polución de la basura del Pacífico tomando muestras. 
En un estudio del 2001, los investigadores (incluyendo a Moore) encontraron que en ciertas áreas del océano, las concentraciones de plástico se acercaban a un millón por milla cuadrada. El estudio encontró concentraciones de plástico de 3,3 partes con una masa de 5,1 miligramos por metro cuadrado. En muchas áreas de la región afectada la concentración de plástico fue mayor a la concentración de zooplancton con un factor de siete. Las muestras tomadas en el fondo de las columnas de agua revelaron niveles bajos de desechos plásticos (primariamente monofilamentos e hilos de pesca), confirmando las primeras impresiones que decían que la principal concentración de desechos estaba en la superficie del mar.

\subsection{IMPACTO EN LA VIDA MARINA}

Los efectos del plástico empiezan en los primeros eslabones de la cadena alimentaria ya que se ha constatado que las medusas los ingieren, de la misma manera que lo hacen peces; las tortugas confunden las bolsas de plástico con medusas (fuentes de agua dulce para estos animales), aves y mamíferos. Se han constatado la muerte por inanición de pollos de albatros en la isla de Midway al ser alimentados por sus padres con material plástico.

Figura N. ${ }^{\circ} 21$

Diferencias entre una bolsa plástica y una medusa marina

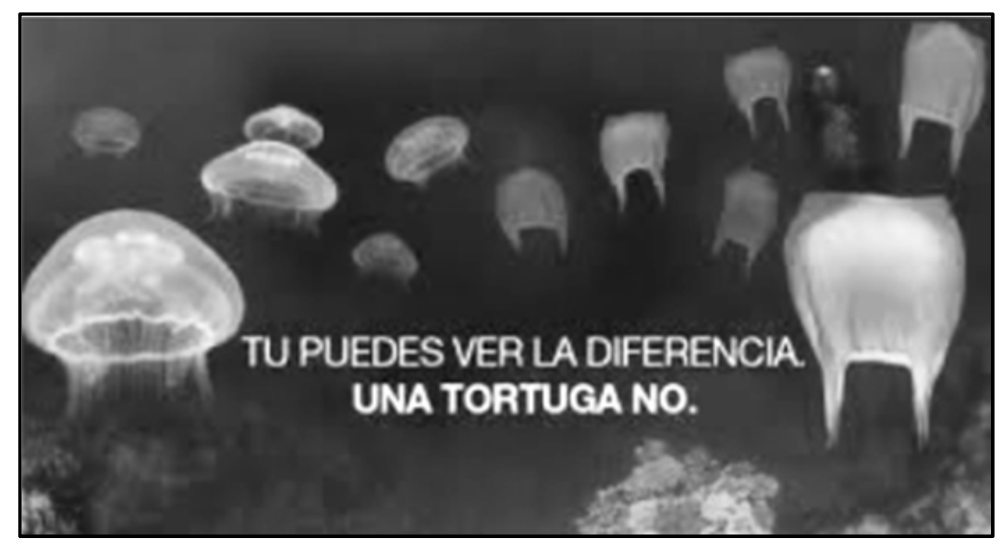

784 Ciencia y Sociedad 2013; 38(4): 743-792 
Además de la ingestión de los residuos, se producen fenómenos de asfixia en aves, peces, mamíferos y reptiles al quedarse estos enredados en los residuos. El PNUMA (Programa de las Naciones Unidas para el Medio Ambiente) asegura que más de 100.000 tortugas y mamíferos marinos mueren cada año como consecuencia de los restos de plástico; y en el anuario de 2011 de la Organización se manifiesta que la contaminación por plásticos en el océano es uno de los tres principales problemas medioambientales emergentes a nivel mundial. Según las Naciones Unidas, el plástico forma parte de la dieta de la mitad de las especies de aves marinas.

Muchos desechos de larga duración terminan en los estómagos de las aves marinas y animales del mar, incluyendo tortugas del océano y albatros de patas negras, siendo estas partículas un riesgo para la vida marina. Por último, el plástico facilita el movimiento de especies invasoras al quedarse estas adheridas a él y ser este relativamente ligero y por ello fácilmente arrestable.

\subsection{EFECTOS QUÍMICOS SOBRE LA VIDA MARINA}

Durante mucho tiempo se ha asegurado que el plástico era inerte y que no podía darse una migración de contaminantes de un medio a otro, pero esto no es cierto. Se ha constatado que la migración de compuestos forma parte de los plásticos a los ecosistemas marinos.

La degradación del plástico se inicia con la fragmentación física del mismo. Como los desechos plásticos flotantes fotodegradables se convierten en trozos más pequeños cada vez, se concentran en la parte superior hasta que se desintegran. El plástico al final llega a ser de un tamaño tan pequeño que puede ser comido por los organismos marinos que viven cerca de la superficie del océano, cosa que favorece su entrada en la cadena alimentaria de los seres vivos por completo. Además debido a que el plástico es poco 
denso, su capacidad de dispersión es muy grande. La degradación del plástico es un proceso complejo ya que bajo el término genérico de plástico se encuentra una serie de compuestos diferentes que responden de manera distinta a los factores ambientales, por ejemplo, algunos son fotodegradables, pero otros no lo son.

Respecto a los diferentes tipos de plástico se ha encontrado ausencia de PET, la razón podría ser que este se han hundido, ya que la densidad de este tipo de plástico es ligeramente mayor a la del agua marina. Los más abundantes serían el polipropileno y el polietileno. Se encontró una gran cantidad de nitrógeno que se atribuye a la acción de algún ser vivo que bien pudo haber utilizado el plástico como soporte, o bien pudo estar alimentándose de él, pero se trata simplemente de conjeturas.

De todas formas, las micropartículas de plástico se absorben a partir del aparato digestivo y se acumulan en animales y personas.

Katsuhiko Saido de la Universidad Japonesa de Chiba ha estudiado la degradación de los plásticos y ha constatado que aquellos que se encuentran en la superficie marina expuestos a la acción de la lluvia y del sol, pueden degradarse de una forma relativamente rápida; la fotodegradación produce cada vez fragmentos más pequeños hasta que se llega al nivel molecular.

Se ha comprobado que la degradación del plástico genera varias sustancias contaminantes, entre ellas bisfenol A, oligómeros de base poliestereno, pero junto a los compuestos fruto de la descomposición de los plásticos se encuentran otros compuestos químicos peligrosos, entre los que pueden destacarse: bifenilos policlorados (PCB), DDT (1,1,1-Tricloro-2,2-bis(4-clorofenil)etano) e Hidrocarburos policíclicos aromáticos (PAH). Saido habla de concentraciones de 150 millones de ppm para algunos compuestos químicos procedentes de la degradación del plástico. 
Investigadores han demostrado que estos residuos plásticos afectan por lo menos a 267 especies alrededor del mundo, la gran mayoría residente en la gran mancha de basura del Pacífico Norte. ${ }^{24}$

\subsection{DAÑOS IRREVERSIBLES}

Los daños en el ecosistema son irreversibles. La basura ha destrozado un tercio de la superficie de hábitats marinos según Naciones Unidas, y se estima que el 35\% de los ecosistemas marinos críticos han sido destruidos. "El desconocimiento, por su inaccesibilidad, ha sido uno de los grandes problemas para la protección de los océanos", según Josep María Gili, biólogo del CSIC, del Instituto de Ciencias del Mar de Barcelona.

Los océanos abarcan más del 90\% de la superficie habitable de la tierra y el 50\% del oxígeno lo producen algas microscópicas -el fitoplancton-, la base de la cadena trófica marina. Los expertos apuntan a que la biodiversidad está en riesgo por un modelo de consumo y producción que no la valora y produce sobreexplotación.

\subsection{POSIBILIDADES DE LIMPIEZA}

En el año 2008, Richard Owen, un contratista de construcción e instructor de buceo, formó la Environmental Cleanup Coalition (Coalición para la Limpieza del Ambiente) para unirse a la causa contra la polución del Pacífico Norte. El FCC (por sus siglas en inglés) planea la modificación de una flota de barcos para limpiar la zona de los desechos como una forma de restauración y reciclaje. El laboratorio creado con este fin se llamó Gyre Island.

La comisaría de la UE, María Damanaki ha propuesto un plan para que la flota pesquera limpie de basura el Mediterráneo, lo

${ }^{24}$ http://www.infolaso.com/continentes-por-superficie.html 
cual, además, representaría una ayuda a la crítica situación del sector; el plástico recogido, pues, podría ser reciclado en plantas especiales.

Richard Owen (en 2008) creó la Environmental Cleanup Coalition para investigar los métodos de mitigación y limpieza del mar.

Retirar los desechos de plástico de los espacios marinos implicaría un coste elevadísimo porque se trabaja con toneladas de material tóxico y se requiere tecnología de punta, de embarcaciones y tripulación de carácter especializada.

\section{Referencias bibliográficas}

Barrero Ripoll, A. (2005). Fundamentos y aplicaciones de la mecánica de fluidos. Madrid: McGraw- Hill.

Bulgakov, S. N., \& Skiba, Y. N. (2003). Modelo de circulación de Stommel. México DF: Centro de Ciencia de la Atmósfera, UNAM.

Cengel, Y. A. (2007). Mecánica de fluidos. México: McGraw-Hill.

Chow, V. T. (1994). Hidraulica de canales abiertos. Bogotá: McGraw-Hill.

Daugherty, R. L., \& Franzini, J. B. (1965). Fluids mechanics with engineering applications (6ta. Ed.). New York: McGraw-Hill.

Debler, W. R. (1990). Fluid mechanics fundamentals. New Jersey: Wiley.

Diccionario de Ciencias de la Tierra. (2000). Madrid: OxfordComplutense. 
Fox, R. W. (1985). Introduction to fluids mechanics (3ra. ed.). New Jersey: Wiley.

Franzini, J. B. (1974). Ingeniería de los recursos hidráulicos. México: CECSA.

Giles, R. V. (1962). Mecánica de los fluidos e bidráulica (2da. Ed.). McGraw-Hill (Compendios Schaum).

Hunsaker, J. C., \& Rightmire, B. G. (1947). Engineering aplications of fluids mechanics. New York: McGraw-Hill.

Hunsaker, J. C., \& Rightmire, B. G. (1986). Aplicaciones prácticas de la mecánica de fluidos en la ingeniería (6ta. Ed.). McGraw-Hill.

John, J. E. (s.f.). Introduccion to fluid mechanics (10ma. Ed.). New Jersey: Prentice-Hall.

Manual de Instrucción de la Armfield . (2009). Londres: Armfield.

Mott, R. L. (2006). Mecánica de fluidos (6ta. Ed.). New York: McGraw-Hill.

Munson, B. R. (1999). Fundamentals of fluids mechanics. New York: John Wiley \& Sons.

Pinilla, M., Villanueva, J., Segura, A., Giraldo, J., \& Jácome, C. (2012). Evolución y comportamiento de la Espiral y Capa Ekman en Superficies de Flujos. Revista Colombiana de Fisica, 44(1).

Potter, M. C. (2005). Mecánica de fluidos (3ra. Ed.). México: Thomson.

Roberson, J. A. (1996). Engineering fluid mechanics (6ta. Ed.). New Jersey: Wiley. 
Shames, I. H. (1995). Mecánica de fluidos (3ra. Ed.). New York: McGraw-Hill.

Smits, A. J. (2005). Una introducción física a la mecánica de fluidos. Santo Domingo: Alfa y Omega.

Sotelo Avila, G. (1995). Fundamentos de bidráulica general. México: Limusa.

Streeter, V. L. (2000). Mecánica de fluidos. Bogotá: McGraw-Hill.

The U.S. Standar Atmosphere. (1976). Washington D. C.: Government Printing Ofice.

Wark, K., Warner, C. F., \& Davis, W. T. (1998). Air pollution: Its origin and control (3ra. Ed.). Boston: Addison-Wesley. 


\section{Martín Antonio Meléndez Valencia}

Ingeniero Civil; maestría en Ciencias de la Construcción para Regiones Tropicales en Desarrollo, y un Post grado en Prevención de Desastres. Trabajo por más de 20 años en Post Desastres siempre con un enfoque holístico y de cuidado medio ambiental. Desde 2007 es docente de la Escuela de Ingeniería del Instituto Tecnológico de Santo Domingo y desde el 2010, encargado de los Laboratorios de Hidráulica y Mecánica de Fluidos.

Email: martin.melendez@intec.edu.do

\section{Patricia Isabel Meléndez Torres}

Ingeniera Civil; maestría en Ingeniería Sanitaria y Medio Ambiente (pendiente tiene la tesis de postgrado). Trabaja en el Ministerio de Obras Públicas desde el año 2009. Es docente invitada al Instituto Tecnológico de Santo Domingo como profesora de práctica de Mecánica de Fluidos.

Email: patty.melendez@gmail.com 
